\title{
Let-7d inhibits intratumoral macrophage M2 polarization and subsequent tumor angiogenesis by targeting IL-13 and IL-10
}

\section{Boxing Su}

Department of Urology, Beijing Tsinghua Changgung Hospital, School of Clinical Medicine, Tsinghua University, Beijing 102218 PR., China.

\section{Haibo Han}

Key Laboratory of Carcinogenesis and Translational Research (Ministry of Education), Department of Clinical Laboratory, Peking University Cancer Hospital \& Institute, Beijing 100142, China.

\section{Yanqing Gong}

Department of Urology, Peking University First Hospital \& the Institute of Urology, Peking University, Beijing 100034 PR., China. National Urological Cancer Center, Beijing 100034 PR., China.

\section{Xuesong Li}

Department of Urology, Peking University First Hospital \& the Institute of Urology, Peking University, Beijing 100034 PR., China. National Urological Cancer Center, Beijing 100034 PR., China.

\section{Chaoyue Ji}

Department of Urology, Beijing Tsinghua Changgung Hospital, School of Clinical Medicine, Tsinghua University, Beijing 102218 PR., China.

\section{Jingjing Yao}

Department of Pathology, Beijing Tsinghua Changgung Hospital, School of Clinical Medicine, Tsinghua University, Beijing 102218 PR., China.

\section{Jianghui Yang}

Department of Pathology, Beijing Tsinghua Changgung Hospital, School of Clinical Medicine, Tsinghua University, Beijing 102218 PR., China.

\section{Weiguo Hu}

Department of Urology, Beijing Tsinghua Changgung Hospital, School of Clinical Medicine, Tsinghua University, Beijing 102218 PR., China.

\section{Wei Zhao}

Key Laboratory of Carcinogenesis and Translational Research (Ministry of Education), Department of Cell Biology, Peking University Cancer Hospital \& Institute, Beijing 100142, China.

\section{Jianxing Li}

Department of Urology, Beijing Tsinghua Changgung Hospital, School of Clinical Medicine, Tsinghua University, Beijing 102218 PR., China.

\section{Gang Zhang ( $\square$ haibian76@163.com )}


Department of Urology, Beijing Tsinghua Changgung Hospital, School of Clinical Medicine, Tsinghua University, Beijing 102218 PR., China

\section{Liqun Zhou}

Department of Urology, Peking University First Hospital \& the Institute of Urology, Peking University, Beijing 100034 PR., China. National Urological Cancer Center, Beijing 100034 PR., China.

\section{Research}

Keywords: microRNA, let-7d, macrophage, polarization, angiogenesis, IL-10, IL-13

Posted Date: May 18th, 2020

DOI: https://doi.org/10.21203/rs.3.rs-27516/v1

License: (9) This work is licensed under a Creative Commons Attribution 4.0 International License. Read Full License

Version of Record: A version of this preprint was published at Cancer Immunology, Immunotherapy on November 25th, 2020. See the published version at https://doi.org/10.1007/s00262-020-02791-6. 


\section{Abstract}

Background: The microRNA let-7d has been reported to be a tumor suppressor in many types of cancer, including renal cell carcinoma (RCC). Tumor-associated macrophages are M2-polarized macrophages that can enhance tumor growth and angiogenesis in many human cancers. However, the role of let-7d in TAM-associated RCC tumor progression remains elusive.

Methods: Human macrophage cell line THP-1 cells pretreated with phorbol myristate acetate were cocultured with let-7d overexpressing RCC cells, the conditioned medium were obtained and its effect on human umbilical vein endothelial cells (HUVECs) was accessed in vitro. The phenotype of cocultured macrophages and macrophages in mouse models bearing let-7d overexpressing RCC cell derived xenograft were analyzed in vitro and in vivo using flow cytometry and quantitative real-time PCR.

Results: First, we observed a strongly inverse correlation between let-7d expression and microvessel density in RCC tissues. Furthermore, the proliferation, migration, and tube formation of HUVECs was significantly inhibited by conditioned medium from a coculture system of the human macrophage THP-1 cell line with let-7d-overexpressing RCC cells. Moreover, the proportion of M2 macrophages was significantly lower in the group that was cocultured with let-7d-overexpressing RCC cells. Subcutaneous xenografts formed by the injection of let-7d-overexpressing RCC cells together with THP-1 cells resulted in a significant decrease in the $\mathrm{M} 2$ macrophage ratio and microvessel density compared with those formed by the injection of control RCC cells with THP-1 cells. In silico analysis revealed interleukin-10 (IL-10) and IL-13 as potential let-7d target genes. Consistently, the activities of the luciferase reporters containing the 3 '-UTRs of the IL-10 and IL-13 genes were significantly inhibited by let-7d. Importantly, the addition of IL10 and IL-13 counteracted the inhibitory effects of the conditioned medium from the coculture system of let-7d-overexpressing RCC cells in vitro. Additionally, the overexpression of IL-10 and IL-13 reversed the effects of let-7d on macrophage M2 polarization and tumor angiogenesis in vivo. Finally, the expression of IL-10 and IL-13 were inversely correlated with the expression of let-7d in RCC clinical specimens.

Conclusions: These results suggest that let-7d may inhibit intratumoral macrophage M2 polarization and subsequent tumor angiogenesis at least in part by targeting IL-10 and IL-13.

\section{Background}

Renal cell carcinoma (RCC) has been described as the most common malignant tumor of the adult kidney and is responsible for approximately $90 \%$ to $95 \%$ of neoplasms that arise from the kidney [1]. Localized RCC, which accounts for approximately two-thirds of RCC cases, can be treated with radical or partial nephrectomy, but the prognosis is poor once the disease advances. The therapeutic options for these patients are limited because conventional radiation therapy and chemotherapy are ineffective, and only 10 to $20 \%$ of these patients benefit from immunotherapy [2].

Tumor angiogenesis has been indicated as a promising therapeutic target for advanced RCC. Targeted drugs that inhibit angiogenesis, such as sunitinib and sorafenib, have an established role in the clinical 
treatment of RCC [3]. Angiogenesis is a complex process that involves multiple steps, including endothelial cell proliferation and migration, matrix degradation and capillary sprouting, all of which are regulated by countervailing factors that either induce or oppose angiogenesis. The well-known inducers of angiogenesis include factors such as vascular endothelial growth factor (VEGF), basic fibroblast growth factor (bFGF), placental growth factor (PIGF) and platelet-derived growth factor (PDGF) [4].

It has long been recognized that the function of cells belonging to the monocyte-macrophage lineage can be profoundly affected by exposure to different microenvironmental signals [5]. Macrophage activation in response to lipopolysaccharide (LPS) and interferon- $\gamma$ (IFN- $\gamma$ ) leads tothe M1 phenotype, and these M1 macrophages exhibit elevated expression of tumor necrosis factor a (TNF-a) and increased ability to kill pathogens and cells [6]. In contrast, the M2 activation of macrophages can be induced by stimuli such as macrophage colony stimulating factor (MCSF), interleukin-4 (IL-4), IL-13, or IL-10 [7]. M2 macrophages exhibit reduced secretion of pro-inflammatory cytokines, such as TNF a, IL-1 $\beta$, and IL-6, and produce high levels of anti-inflammatory cytokines, such as transforming growth factor- $\beta$ (TGF- $\beta$ ) [8]. Tumorassociated macrophages (TAMs) represent a major population of the leukocytes that infiltrate tumors, and TAMs are driven by tumor- and T cell-derived cytokines to undergo polarization toward the M2 phenotype [9]. TAMs are known to promote tumor progression and metastasis by enhancing tumor growth, angiogenesis, and suppression of adaptive immunity [10]. A high density of tumor-infiltrating TAMs has been demonstrated to be associated with a poor prognosis in various types of cancer, including RCC [11, 12]. Furthermore, the depletion of TAMs enhances the sorafenib-induced inhibition of tumor progression and angiogenesis [13].

Let-7 was among the first microRNAs to be identified and was originally discovered in the nematode Caenorhabditis elegans. Let-7 consists of 13 family members that are highly conserved across species [14]. Let-7 is frequently downregulated in many human malignancies, suggesting its pivotal role in oncogenesis $[15,16]$. Previously, we found that let-7d expression was significantly downregulated in RCC. Decreased let-7d expression was associated with advanced T stages and increased microvessel density in RCC patients. Furthermore, high densities of macrophage infiltration were observed in RCC and were inversely correlated with let-7d expression [17]. Considering the important role of TAMs in tumor angiogenesis, the current study was designed to investigate whether let-7d functionally participates in macrophage polarization and, thus, plays a role in tumor angiogenesis in RCC.

\section{Materials And Methods}

\section{Clinical samples}

Histologically confirmed RCC tissues and paired adjacent normal tissue were collected from 80 patients who underwent radical nephrectomy at Peking University First Hospital between 2012 and 2013; after collection, the tissues were snap-frozen in liquid nitrogen. The Ethics Committee of Peking University First Hospital approved this study. Written informed consent was obtained from all the patients.

\section{Cell lines and reagents}


Human RCC cell lines 769P and 7860 were purchased from ATCC (MA, USA). The human acute monocytic leukemia cell line THP-1 and human umbilical vein endothelial cells (HUVECs) were obtained from the China Center for Type Culture Collection (Wuhan, China). Pri-let-7d lentivirus stably transfected 7860 and 769P cell lines overexpressing let-7d, as well as the corresponding vehicle-transfected control cells, were established as previously described [17]. The transformed human embryonic kidney 293FT cells were purchased from Invitrogen. The 7860 and 769P cells were maintained in RPMI 1640 medium. The HUVECs were maintained in DMEM/F12 (50:50, v/v) medium. The 293FT cells were routinely cultured in DMEM. All the culture media were supplemented with $10 \%(\mathrm{v} / \mathrm{v})$ fetal bovine serum (FBS), 100 $\mathrm{U} / \mathrm{ml}$ penicillin sodium and $100 \mu \mathrm{g} / \mathrm{ml}$ streptomycin sulfate, and the cells were incubated at $37^{\circ} \mathrm{C}$ in a standard humidified incubator containing $5 \% \mathrm{CO}_{2}$.

Phorbol-12-myristate-13-acetate (PMA) was purchased from Sigma-Aldrich. THP-1 cells were seeded at 2 $\times 10^{5}$ cells $/ \mathrm{cm}^{2}$ and treated with PMA $(200 \mathrm{nM})$ for $48 \mathrm{~h}$ to differentiate the cells into resting macrophages (M0 cells) as previously described [18].

\section{In vivo assay}

Approval of the animal studies was obtained from the Ethics Committee of Tsinghua Changgung Hospital. Specific pathogen-free (SPF) 5-week-old female BALB/C-nu/nu nude mice (Vital River Laboratory Animals, Beijing, China) were used in this study and maintained in accordance with institutional guidelines for the use of laboratory animals. A total of $3 \times 10^{6} 7860$ cells stably transfected with lentivirus and $3 \times 10^{6}$ THP- 1 cells treated with PMA (M0) were subcutaneously injected into the right flank of each mouse in a 1:1 mixture of Matrigel (BD Biosciences, San Jose, CA). Five mice were included in each group. After 7 weeks, the mice were euthanized, and the tumors were dissected, weighed and prepared for RNA isolation.

\section{RNA extraction and quantitative real-time polymerase chain reaction (qRT-PCR)}

Total RNA was extracted using the miRNeasy Mini Kit (Qiagen, Hilden, Germany) according to the manufacturer's instructions. To analyze the mature miRNA, $1 \mu \mathrm{g}$ total RNA was polyadenylated to add a polyA tail with polyA polymerase (NEB, Beverly, MA, USA) and then reverse-transcribed with an oligo-dT adapter primer into cDNA for qRT-PCR quantification [17]. To detect the protein-coding mRNA, cDNAs were synthesized from $2 \mu \mathrm{g}$ of total RNA using Moloney murine leukemia virus reverse transcriptase and oligo(dT) ${ }_{15}$ primers (Invitrogen). Quantitative RT-PCR was performed using the SYBR Select Master Mix (Life) in a final volume of $10 \mu \mathrm{L}$ on an ABI7500 Fast PCR machine (Applied Biosystems, Foster City, CA, USA). U6 and GAPDH were selected as the endogenous references for miRNA and mRNA, respectively. Relative quantification (RQ) was calculated based on the $2^{-\Delta \mathrm{Ct}}$ method, where $\Delta \mathrm{Ct}=\mathrm{Ct}$ (target) ${ }^{-C t}$ (reference). The fold change was calculated by the $2^{-\Delta \Delta c t}$ method. For a list of all the primers, see Supporting Information, Table S1.

\section{Lentiviral vector construction, virus packaging and cell infection}


All the constructs were generated by standard DNA recombination techniques as previously described [17]. Briefly, the open reading frames of the IL-10 and IL-13 cDNAs were obtained by RT-PCR and cloned into the vector pcDNA3.1. All of the constructs were verified by sequencing. The sequences of the primers are listed in Table S1 (see Supporting Information). Lentiviruses were produced using the ViraPower ${ }^{\mathrm{TM}}$ Packaging Mix (Invitrogen) in 293FT packaging cells. The virus-infected cells were selected with $5 \mu \mathrm{g} / \mathrm{ml}$ blasticidin $+500 \mu \mathrm{g} / \mathrm{ml} \mathrm{G} 418$ (Invitrogen). The antibiotic-resistant clones were pooled and used for the subsequent assays.

\section{In vitro cell coculture and conditioned medium preparation}

RCC cells were seeded in 6-well plates at a density of $1 \times 10^{5}$ cells/well in serum-containing medium. Twenty-four hours later, the medium was replaced with serum-free RPMI 1640 medium, and the cells were incubated for an additional $48 \mathrm{~h}$. The conditioned medium was collected and centrifuged at $3000 \times \mathrm{g}$ for 5 min to remove the cells or cell debris.

Transwell cellculture inserts (Corning Incorporated, Corning, NY) with 12-mm polyester (PET) membranes (pore size: $0.4 \mu \mathrm{M}$, density: $1.6 \times 10^{6}$ pores $/ \mathrm{cm}^{2}$ ) were used for the noncontact coculture of RCC cells and THP-1 cells in 6-well plates, as previously described [19]. Briefly, $2 \times 10^{5}$ PMA-pretreated TPH-1 cells were cocultured with RCC cells in serum-free RPMI 1640 medium at $37^{\circ} \mathrm{C}$ in an atmosphere containing $5 \% \mathrm{CO}_{2}$. Twenty-four hours later, the culture medium from the lower compartments was collected, centrifuged and used as conditioned medium. In the in vitro rescue experiment, human recombinant IL-10 and IL-13 (R\&D Systems, Minneapolis, MN) were added to the lower chamber of the coculture system at a concentration of $100 \mathrm{ng} / \mathrm{mL}$ and incubated for $24 \mathrm{~h}$ before collecting the conditioned medium.

\section{In vitro cell proliferation, migration, and tube formation assay}

HUVECs were seeded in 96-well plates $\left(5 \times 10^{3}\right.$ cells/well) and cultured in conditioned medium from RCC cells alone or THP-1 cells cocultured with RCC cells. Cell proliferation was evaluated by a Cell Counting Kit-8 (CCK-8, Dojindo, Kumamoto, Japan) at various time points for up to four days according to the manufacturer's instructions. The cell migration assays were performed using a Boyden chamber as previously described [17]. Briefly, $2 \times 10^{5}$ HUVECs in $100 \mu$ were added to the upper chamber of the 24transwell apparatus, and $800 \mu \mathrm{l}$ conditioned medium was added to the lower chamber. After $24 \mathrm{~h}$ of incubation, the cells that migrated though the membrane were fixed with $100 \%$ methanol, stained with $0.1 \%$ crystal violet stain for $5 \mathrm{~min}$, and counted under a microscope. Five high-power fields (magnification, 200x) per well were randomly selected and manually counted. The Fibrin In Vitro Angiogenesis Assay (Millipore) was used to analyze new blood vessel formation according to the manufacturer's instructions. Briefly, $96-$ well plates were coated with cold liquid ECMatrix ( $50 \mu \mathrm{L} /$ well) and incubated at $37^{\circ} \mathrm{C}$ for $1 \mathrm{~h}$. Then, HUVECs were seeded at a density of $5 \times 10^{3}$ cells/well on the polymerized ECMatrix and incubated with conditioned media at $37^{\circ} \mathrm{C}$ for 6 to $8 \mathrm{~h}$. Branch number was observed under a phase-contrast microscope. The enclosed networks in 5 random fields per well were measured by Image J software, and the average value was calculated. The experiment was repeated in triplicate. 


\section{Immunohistochemistry (IHC)}

Paraffin-embedded tissues were analyzed using immunohistochemical staining with an anti-CD31 antibody (DAKO, Carpinteria, CA) and the microvessel density (MVD) was evaluated as previously described [20]. CD31 was used as a vascular endothelial cell marker. Any morphologically identifiable vessels with a lumen and CD31-positive endothelial cell clusters that were clearly separate from the adjacent microvessels in the selected field were all counted as individual vessels. Five independent areas with the highest numbers of discrete microvessels were selected, digitally photographed, and manually counted under a microscope using a 20x objective lens (Olympus IX71, Olympus Optical Co. Ltd, Tokyo, Japan). The average vessel counts for each tumor tissue were used for the statistical analysis. Two pathologists who were blinded to the patients' clinical data independently examined all the slides.

\section{ELISA}

The conditioned medium from the cocultured M0 cells was used to detect PIGF by a ELISA kit (R\&D Systems, Minneapolis, MN) according to the manufacturer's protocol. The optical density (OD) was measured at $450 \mathrm{~nm}$ with a Multiskan microplate spectrophotometer (ThermoLab Systems, Milford, MA).

\section{Western blot analysis}

Total protein was extracted from the tumor tissues and cultured cells using a radioimmunoprecipitation assay (RIPA) kit (ShineGene Molecular Biotech, Inc., Shanghai, China), as instructed by the manufacturer. After determining the protein concentration using a bicinchoninic acid (BCA) kit (Pierce, USA), equal amounts of the total protein were subjected to $10 \%$ SDS polyacrylamide gel electrophoresis (SDS-PAGE). Thereafter, the proteins were blotted onto polyvinylidene difluoride (PVDF) membranes (Pall, Pensacola, $\mathrm{FL})$. After the nonspecific binding sites were blocked, the blots were incubated with antibodies against IL10 or IL-13 (Abcam, Cambridge, UK) for $1 \mathrm{~h}$ at $37^{\circ} \mathrm{C}$. After three washes with TBST and incubation for $2 \mathrm{~h}$ with the appropriate HRP-conjugated secondary antibodies (Applygen, China), the Immobilon ${ }^{\mathrm{TM}}$ Western Chemiluminescent HRP substrate (Millipore) was used to detect the proteins of interest on the blots. The blotted membranes were then scanned using GeneSnap (Syngene, Cambridge, UK) acquisition software.

\section{Flow cytometry}

Cells were centrifuged at $1500 \mathrm{rpm}$ for $5 \mathrm{~min}$, washed with PBS and filtered through a 100- $\mu \mathrm{m}$ mesh for flow cytometry. The cells were subsequently stained with an antibody against an M2 macrophage surface markers (PE-labeled anti-human CD204 (R\&D Systems, Minneapolis, MN)) for 30 min at $4{ }^{\circ} \mathrm{C}$. Isotype control antibodies (Biolegend) were used as negative controls. The cells were analyzed using a flow cytometer (FAC-SCalibur, BD Biosciences). The collected data were processed by the CellQuest program (BD Biosciences). The experiment was repeated in triplicate.

\section{Dual luciferase activity assay}


The 3'-untranslated regions (UTRs) of human IL-10 and IL-13 containing putative binding sites of let-7d and the corresponding mutant binding sites were chemically synthesized and inserted immediately downstream of the firefly luciferase cDNA in the pGL3-control vector (Promega, Madison, WI) by GenePharma (Shanghai, China) to generate the pGL3-IL-10, pGL3-IL-13, pGL3-IL-10-Mut, and pGL3IL-13-Mut constructs. 293FT cells were plated at $1.5 \times 105$ cells/well in 24-well plates $24 \mathrm{~h}$ before the transfection.ThepGL3 constructs $(0.3 \mu \mathrm{g})$ plus the pRL-TK plasmid that expressed Renilla luciferase (26 ng; Promega) were transfected in combination with $60 \mathrm{pmol}$ of either a stability-enhanced non targeting oligonucleotide control or let-7d mimics (GenePharma, Shanghai, China) using Lipofectamine 2000 (Invitrogen). Twenty-four hours after transfection, the luciferase activity was measured using the Dual Luciferase Reporter Assay System (Promega) on the Varioskan ${ }^{T M}$ Flash Multimode Reader (Thermo Fisher Scientific, Inc., Waltham, MA) according to the manufacturer's instructions. For each transfected well, the firefly luciferase activity was normalized to the Renilla luciferase activity. The results were obtained from three independent experiments, and each experiment was performed in quadruplicate.

\section{Statistical analysis}

All the data are presented as the mean \pm SD of at least three independent experiments and were analyzed using SPSS 20.0 statistical software (IBM, Chicago, IL, USA). The significance of the difference between two groups was determined using double-sided Student's t-test. In the case of multiple tests, one-way ANOVA followed by the Bonferroni-Holm procedure was used unless otherwise specified. Correlations were evaluated using two-tailed Spearman's test. $p<0.05$ was considered statistically significant.

\section{Results}

\section{The conditioned medium from let-7d-overexpressing RCC cells had no effects on the proliferation, migration and tube formation of HUVECs}

To investigate the role of let-7d expression in RCC cells on tumor angiogenesis, we evaluated the association between let-7d expression and microvessel density (MVD) in 80 RCC tissues. A strong inverse correlation between let-7d expression and MVD was observed (Spearman's $r=-0.40, p<0.001$ ) (Fig. S1). This finding suggests a regulatory role of let-7d in tumor angiogenesis. To further investigate the underlying mechanism, we used conditioned medium from the RCC cell lines 7860 and 769P stably transfected with pri-let-7d lentivirus and measured its effects on the proliferation, migration and tube formation of the immortalized human umbilical endothelium cell line HUVEC in vitro. Interestingly, as shown in Fig. 1, the proliferation, migration and tube formation of the HUVECs cultured with conditioned medium from the lenti-let-7d-transfected 7860 and 769P cells were not significantly different from those of the HUVECs cultured with conditioned medium from the vehicle-transfected control cells. These results demonstrated that the restoration of let-7d in RCC cells did not influence the proliferation, migration and tube formation of HUVECs in vitro. Considering the strong inverse relationship between let-7d expression and MVD in tumor tissues, our data suggested that let-7d expression in RCC cells might play an indirect role in tumor angiogenesis. 


\section{The conditioned medium from THP-1 cells cocultured with let-7d-overexpressing RCC cells inhibited the proliferation, migration, and tube formation of HUVECs}

Increasing evidence has demonstrated that the interplay between cancer cells and neoplastic stromal cells is of great importance to tumor initiation and progression [21]. Tumor-associated macrophages are important stromal cells that are present in high numbers in the tumor microenvironment and have been shown to preserve many tumor-promoting functions, including suppression of antitumor immune responses and promotion of metastatic growth and tumor angiogenesis [10]. Our previous study showed that significantly higher numbers of macrophages, than other stromal cells, infiltrated RCC tissues, and this infiltration was inversely correlated with let-7d expression [17]. To investigate whether macrophages are involved in let-7d-related tumor angiogenesis in RCC, we employed a noncontact cell coculture system. The PMA-treated macrophage cell line THP-1 was cocultured with let-7d-overexpressing or control RCC cells. The culture medium was then collected from the THP-1 cells and used as conditioned medium. As shown in Fig. 2, the proliferation, migration and tube formation of HUVECs cultured with conditioned medium from the THP-1 cells cocultured with let-7d-overexpressing 7860 and 769P cells were significantly decreased compared with those of HUVECs cultured with conditioned medium from the THP-1 cells cocultured with control 7860 and 769P cells. Taken together, these data suggested that let-7d expression in RCC cells could influence the proliferation, migration and tube formation of HUVECs through the macrophage THP-1 cell line, indicating the important role of macrophages in let-7d-related tumor angiogenesis in RCC.

\section{Let-7d overexpression in RCC cells inhibited the M2 polarization of THP-1 cells cocultured with RCC cells}

Macrophages have been reported to have a high degree of functional plasticity depending on the different microenvironmental signals to which they are exposed. Macrophages that infiltrate tumor tissue can polarize toward the M2 phenotype, and these M2 macrophages are oriented toward promoting tumor growth and facilitating angiogenesis [10]. To investigate whether the PMA-treated THP-1 cells (M0) cocultured with RCC cells polarized into the M2 phenotype and therefore affected the process of angiogenesis, we employed flow cytometry analysis to evaluate the percentage of M2 macrophages among the $\mathrm{M0}$ macrophages cocultured with let-7d-overexpressing 7860 and 769P cells. CD204 was used as an M2 surface macrophage marker [22]. As shown in Fig. 3A and B, the percentage of CD204positive cells was lower in the M0 cells cocultured with let-7d-overexpressing RCC cells than in those cocultured with the corresponding control RCC cells. We next evaluated the cytokine profiles of the M0 cells cocultured with let-7d-overexpressing or control RCC cells to determine whether they possess M2 characteristics. As shown in Fig. $3 \mathrm{C}$ and $\mathrm{D}$, the expression levels of the proinflammatory cytokine TNF- $\mathrm{a}$ was significantly lower in the M0 cells cocultured with control 7860 and 769P cells compared with theM0 cells cocultured with let-7d-overexpressing 7860 and 769P cells. The anti-inflammatory cytokine TGF- $\beta$ was significantly higher in the M0 cells cocultured with control 7860 and 769P cells compared with the M0 cells cocultured with let-7d-overexpressing 7860 and 769P cells. Taking the surface marker and 
cytokine profiles together, the results showed that let-7d overexpression inhibited the RCC cell cocultureinduced M2 polarization of the PMA-treated THP-1 macrophages. Since M2 macrophages have been reported to promote tumor angiogenesis, we evaluated whether let-7d expression in cocultured RCC cells influenced the angiogenic cytokine secretion of macrophages. Quantitative RT-PCR screening identified that among angiogenic factors including VEGF, PDGF, PIGF, FGF and MMP-9 (data not shown), only the expression level of PIGF was significantly decreased in the M0 macrophages cocultured with let-7doverexpressing RCC cells (Fig. 3E and F). As shown by ELISA, the protein level of PIGF in conditioned medium from the coculture system with let-7d-overexpressing RCC cells was also decreased. These results indicated that let-7d expression in RCC cells inhibited the M2 polarization of the THP-1 macrophages cocultured with RCC cells and therefore affected the process of angiogenesis.

\section{Let-7d overexpression inhibited macrophage M2 polarization and tumor angiogenesis in vivo}

Next, we used a subcutaneous xenograft model that was established by inoculating 7860 cells together with M0 macrophages into nude mice to investigate the influence of let-7d overexpression on macrophage polarization and tumor angiogenesis in vivo. As shown in Fig. 4A, the microvessel density in the let-7d-overexpressing tumors was significantly decreased compared with that in the control tumors. Consistently, the tumor growth of let-7d-overexpressing groups was also significantly inhibited, and the mean tumor weight was reduced by $83.8 \%$ (Fig. 4B). To investigate the phenotypic differentiation of the macrophages, we employed qRT-PCR to analyze the mRNA expression level of the M2 phenotype marker CD204 and the panmacrophage marker CD68. The ratio of the CD204/CD68 mRNA levels in the let-7doverexpressing groups was significantly lower than that in the control groups (Fig. 4C). Furthermore, a strong inverse correlation was observed between the expression of let-7d and the mRNA level of CD204 in the same set of 80 RCC tissue samples as evaluated by qRT-PCR (Fig. 4D). Taken together, these data suggested that let-7d overexpression inhibited macrophage M2 polarization, tumor growth and angiogenesis.

\section{IL-13 and IL-10 were direct target genes of let-7d in RCC cells}

To investigate the potential mechanisms involved in the suppressive effects of the overexpression of let$7 \mathrm{~d}$ on the M2 polarization of macrophages cocultured with RCC cells, we first performed in silico screening using 3computational algorithms for mRNA target prediction-miRanda (http://miRdb.org/miRDB/index.html), TargetScan (http://www.targetscan.org), and PicTar (https://pictar.mdc-berlin.de/). A list of predicted candidate let-7d target genes was obtained; among these candidate genes, we tried to identify genes that were potentially involved in macrophage polarization through data mining of the Gene Expression Omnibus database (GEO; accession number: GSE781; GSE15641) [23, 24]. Quantitative RT-PCR analysis confirmed that the expression of IL-13 and IL10 was significantly decreased in the pri-let-7d lentivirus-transfected 7860 and 769P cells compared with the control cells (Fig. 5A). The protein levels of IL-13 and IL-10 also decreased after let-7d overexpression, as demonstrated by western blot assay (Fig. 5B-C). These data demonstrated that forced 
expression of let-7d in RCC downregulated the endogenous expression of IL-13 and IL-10 at both the mRNA and protein levels.

To further investigate whether these genes are bona fide targets of let-7d, luciferase reporters containing the wild-type and mutant (Mut) sequences of the putative let-7d binding site within the $3^{\prime}$-UTRs of the two genes were created (Fig. 5D). As shown in Fig. 5E, the luciferase activities were significantly decreased in reporters containing the wild-type 3'-UTRs of these two genes, but the luciferase activities in the corresponding mutant constructs were not decreased. Thus, the let-7d binding sites in the 3'-UTRs of IL13 and IL-10 were responsible for the reduced activities of the luciferase reporters, indicating that let-7d modulated the expression of these two genes by directly binding to their 3'-UTRs. Taken together, these results suggested that IL-13 and IL-10 were direct targets of let-7d.

\section{Rescue of IL-10 and IL-13 eliminated the effects of let-7d}

To confirm that IL-10 and IL-13 are required for the effects of let-7d on macrophage M2 polarization and tumor angiogenesis, we performed rescue experiments by coculturing THP-1 macrophages with RCC cells in the presence of purified IL-10 or IL-13. The addition of $100 \mathrm{ng} / \mathrm{ml} \mathrm{IL-10}$ or IL-13 rescued the M2 polarization of the cocultured THP-1 macrophages respectively, as demonstrated by flow cytometry analysis using CD204 as an M2 phenotype marker (Fig. 6A). Consistently, while the growth, migration and tube formation of HUVECs decreased upon exposure to conditioned medium from the coculture system with let-7d-overexpressing RCC cells, the addition of IL-10 or IL-13 completely eliminated this effect (Fig. 6B-D). Furthermore, the cytokine profiles and the protein level of PIGF in conditioned medium from the THP-1 macrophages cocultured with let-7d-overexpressing RCC cells was also reversed in the presence of IL-10 or IL-13 respectively (Fig. 6E-H). Next, we separately introduced expression cassettes without the 3'UTRs of these genes into let-7d-overexpressing 7860 cells to evaluate the effects of let-7d in vivo. The expression of IL-10 and IL-13 was recovered, and the let-7d-mediated suppression of macrophage M2 polarization, tumor growth, and angiogenesis in the subcutaneous xenograft model was reversed when the let-7d-overexpressing 7860 cells were infected with IL-10- and IL-13-expressing lentiviruses compared with control virus (Fig. 7A-D). These data demonstrated thatlet-7d expression in RCC cells impeded macrophage M2 polarization and tumor angiogenesis by directly targeting IL-10 and IL-13.

\section{Let-7d expression inversely correlated with the IL-10 and IL-13 mRNA levels in RCC tissues}

We then analyzed the expression of let-7d and the mRNA levels of IL-10 and IL-13 by qRT-PCR in the same set of 80 RCC tissues. As shown in Fig. $7 \mathrm{E}-\mathrm{F}$, statistically significant inverse correlations were observed between the expression of let-7d and the mRNA levels of IL-10 and IL-13 in the RCC tissues. These data supported the premise that let-7d downregulation increased the mRNA expression levels of IL10 and IL-13 in clinical specimens of human RCC.

\section{Discussion}


Let-7d has been reported to function as a tumor suppressor in many cancer types [25]. Previously, we found that let-7d suppresses growth, metastasis, and tumor macrophage infiltration in RCC by targeting COL3A1 and CCL7 [17]. In this study, we further demonstrated that let-7d inhibited intratumoral macrophage M2 polarization and the subsequent tumor angiogenesis by targeting interleukin-10 (IL-10) and IL-13. Tumor-associated macrophages (TAMs) are a major component of the RCC tumor microenvironment and contribute to malignant cancer progression by orchestrating various aspects of cancer, such as tumor cell proliferation, invasion, metastasis, angiogenesis and immunosuppression [26]. In RCC, infiltrating macrophages increase cancer stem cell-like populations, and the number of TAMs is highly correlated with the density of tumor microvessels [27, 28]. TAMs significantly contribute to tumor angiogenesis by producing a wide array of growth factors, and by stimulating several crucial signaling pathways, including the VEGF/VEGFR-1 pathway [29].Moreover, VEGF in RCC tumors may be mainly produced by tumor stromal cells, especially macrophages, instead of tumor cells themselves [30]. The reported M2 or TAM markers in the tumor microenvironment include CD68, CD163, CD206, CD204 and CD209 [31, 32]. However, there are some discrepancies in the published literature regarding these markers. Some studies used CD68 or CD163 as a marker of TAMs and demonstrated that increased numbers of CD68- or CD163-positive cells in RCC are associated with poor prognosis [33, 34]. Some observations using an immunohistochemical approach have observed that CD163 cannot be considered a reliable M2 marker when used alone, and CD68 was used as a panmacrophage marker [35, 36]. CD204 has been used as an M2 marker in many studies and has been validated to be associated with tumor aggressiveness and unfavorable prognosis in many cancers [37, 38]. In our study, we used CD204 as a marker of M2 macrophages and found that after coculture with RCC cells, the proportion of CD204positive macrophages was significantly increased, and the cytokine profiles shifted toprofiles that are characteristic of the M2 phenotype. Furthermore, we found that CD204 expression is inversely correlated with let-7d expression in RCC tissues, indicating that the CD204 antigen might be a better marker of the M2 phenotype in RCC tissues.

Interleukin (IL)-10, which was originally called cytokine synthesis inhibitory factor produced by type 2 helper T-lymphocytes (Th2), plays an important role in the negative feedback loop that prevents uncontrolled inflammation and has subsequently been described as a potentially immunosuppressive cytokine [39]. IL-10 has been detected in many types of cancer, and the high expression of IL-10 in the serum has been demonstrated to predict worse outcomes for cancer patients [40]. In RCC, IL-10 has been suggested to play a critical role in generating an immunosuppressive tumor microenvironment and to contribute to reduced tumor-infiltrating T cell effector functions [41]. Accumulating studies have suggested that this immunosuppressive function of IL-10 may be partly related to the M2 polarization of macrophages. IL-10 can polarize tumor-infiltrating macrophages toward the M2 phenotype, and M2polarized macrophages, in turn, secrete more IL-10 [42].In pancreatic cancer, increased IL-10 expression by M2-polarized macrophages promoted the epithelial-mesenchymal transition of cancer cells [43]. In RCC, bone morphogenetic protein-6 (BMP-6) was reported to increase the secretion of IL-10 in tumorinfiltrating macrophages, polarize these macrophages toward the M2 phenotype and consequently promote RCC progression. Furthermore, patients with elevated IL-10 serum levels had worse outcomes 
after surgical treatment of RCC [34]. In our study, IL-10 was identified as a target gene of let-7dand was inversely correlated with the expression of let-7d. Furthermore, the restoration of IL-10 alone in let-7doverexpressing RCC cells increased tumor growth and angiogenesis in vivo, indicating the important role of IL-10 in RCC progression.

IL-13 is a Th2 cytokine that is secreted by a wide variety of different cell types, including Th2-polarized T cells, monocytes/macrophages, granulocytes and cancer cells [44]. IL-13 has been reported to be an antiinflammatory and immunoregulatory cytokine that can suppress tumor immunosurveillance through the activation of interleukin 4 receptor a (IL-4Ra) and its downstream transcriptional factor STAT 6 in natural killer T cells (NKT cells) [45]. IL-13 has also been shown to mediate cancer invasion and metastasis via its receptor, IL-13Ra2, in an ERK/AP-1-dependent and MMP-mediated manner in a mouse model of human ovarian tumor cancer [46]. Moreover, a significant elevation in the plasma levels of IL-13 has been observed in patients with pancreatic, esophageal and gastric cancer [47]. High expression of IL-13 was associated with increased recurrence and reduced survival in localized clear cell RCC, as demonstrated by immunohistochemistry staining [48]. Many studies have validated that the IL-13 secreted by tumor cells or tumor stromal cells can polarize tumor-infiltrating macrophages toward the M2 phenotype, which can be tumor-trophic $[49,50]$. Our study suggests that let-7d can simultaneously target two potent macrophage stimuli, IL-10 and IL-13, by which macrophages can be polarized toward the M2 phenotype and promote tumor angiogenesis, suggesting the potential role of let-7d as a target of immunotherapy for RCC.

In summary, our results indicate that let-7d can inhibit macrophage M2 polarization and tumor angiogenesis, at least in part through the direct destabilization of the mRNAs of IL-10 and IL-13. These findings may improve our understanding of the mechanisms involved in the progression of RCC and the interaction of RCC cells with tumor stromal cells, contributing to the discovery of better prognostic markers and therapeutic targets of RCC.

\section{Conclusions}

This study demonstrated that let-7d inhibits the M2 polarization of tumor-infiltrating macrophages and the subsequent tumor angiogenesis at least in part by targeting IL-10 and IL-13. Our findings suggest let$7 \mathrm{~d}$ as a promising target for RCC immunotherapies.

\section{Abbreviations}

RCC, renal cell carcinoma; qRT-PCR, quantitative reverse transcription-polymerase chain reaction; 3'UTR: 3'-untranslated regions; TAM, tumor-associated macrophage; HUVEC, human umbilical vein endothelial cell; PMA, phorbol myristate acetate; VEGF, vascular endothelial growth factor; FGF, fibroblast growth factor; EGF, epidermal growth factor; PIGF, placental growth factor; PDGF, platelet-derived growth factor;MVD, microvessel density; TNF- $\alpha$, tumor necrosis factor $\alpha$; TGF- $\beta$, transforming growth factor- $\beta$. 


\section{Declarations}

\section{Author contributions}

Design: B.S., H.H., Y.G., W.Z., G.Z and L.Z. Experimental operation: B.Su, H.H, W.H, X.L., J.Yao. and J.Yang. Acquisition of data and Analysis (acquired and managed patients' information and statistical analysis): B.Su, H.H., Y.G., X.L. and G.Z. Writing, review, and/or revision of the manuscript: B.Su, H.H., G.Z. and L.Z. All authors read and approved the submitted manuscript.

\section{Availability of data and materials}

All data supporting the conclusions of this article are included within the article.

\section{Acknowledgements}

The authors thank Yunfei Fan for the support and help for technical assistance.

\section{Funding}

This study was supported by grants from the National Natural Science Foundation (Grant No.8170101445), the Beijing Natural Science Foundation (No.7182191) and the Capital Health Research and Development of Special (code: 2016-1-2241) .

\section{Ethics approval and consent to participate}

All Procedures performed in studies involving human participants or animals were reviewed and granted by the Ethics Committee of Peking University First Hospital and the Ethics Committee of Tsinghua Changgung Hospital, Beijing, China. Informed consent was obtained from all the patients.

\section{Consent for publication}

Not applicable.

\section{Conflicts of Interest}

The authors declare no conflict of interest.

\section{References}

1. Barata PC, Rini BI. Treatment of renal cell carcinoma: Current status and future directions. CA Cancer J Clin. 2017; 67(6): 507-524.

2. Motzer RJ, et al. Overall survival and updated results for sunitinib compared with interferon alfa in patients with metastatic renal cell carcinoma. J Clin Oncol. 2009; 27(22): 3584-90. 
3. McDermott DF, et al. Clinical activity and molecular correlates of response to atezolizumab alone or in combination with bevacizumab versus sunitinib in renal cell carcinoma. Nat Med. 2018; 24(6): 749-757.

4. McMahon G. VEGF receptor signaling in tumor angiogenesis. Oncologist 2000; 5 Suppl 1: 3-10.

5. Mantovani A, et al. : Macrophage polarization: tumor-associated macrophages as a paradigm for polarized M2 mononuclear phagocytes. Trends Immunol. 2002; 23(11): 549-55.

6. Lu G, et al. Myeloid cell-derived inducible nitric oxide synthase suppresses M1 macrophage polarization. Nat Commun. 2015; 6: 6676.

7. Murray PJ, et al. Macrophage activation and polarization: nomenclature and experimental guidelines. Immunity. 2014; 41(1): 14-20.

8. Tjiu JW, et al. Tumor-associated macrophage-induced invasion and angiogenesis of human basal cell carcinoma cells by cyclooxygenase-2 induction. J Invest Dermatol. 2009; 129(4): 1016-25.

9. Erreni M, Mantovani A, Allavena P. Tumor-associated Macrophages (TAM) and Inflammation in Colorectal Cancer. Cancer Microenviron. 2011; 4(2): 141-54.

10. Pollard JW. Tumour-educated macrophages promote tumour progression and metastasis. Nat Rev Cancer. 2004; 4(1): 71-8.

11. Qian BZ, Pollard JW. Macrophage diversity enhances tumor progression and metastasis. Cell 2010; 141(1): 39-51.

12. Xu L, et al. Prognostic value of diametrically polarized tumor-associated macrophages in renal cell carcinoma. Ann Surg Oncol. 2014; 21(9): 3142-50.

13. Zhang W, et al. Depletion of tumor-associated macrophages enhances the effect of sorafenib in metastatic liver cancer models by antimetastatic and antiangiogenic effects. Clin Cancer Res. 2010; 16(13): 3420-30.

14. Boyerinas B, et al. The role of let-7 in cell differentiation and cancer. Endocr Relat Cancer. 2010; 17(1): F19-36.

15. Elghoroury EA, et al. Evaluation of miRNA-21 and miRNA Let-7 as Prognostic Markers in Patients With Breast Cancer. Clin Breast Cancer. 2018; 18(4): e721-e726.

16. Dou R, et al. MicroRNA let-7, T Cells, and Patient Survival in Colorectal Cancer. Cancer Immunol Res. 2016; 4(11): 927-935.

17. Su B, et al. Let-7d suppresses growth, metastasis, and tumor macrophage infiltration in renal cell carcinoma by targeting COL3A1 and CCL7. Mol Cancer. 2014; 13: 206.

18. Miyake M, et al. CXCL1-Mediated Interaction of Cancer Cells with Tumor-Associated Macrophages and Cancer-Associated Fibroblasts Promotes Tumor Progression in Human Bladder Cancer. Neoplasia. 2016; 18(10): 636-646.

19. Desai N, et al. Development of a xeno-free non-contact co-culture system for derivation and maintenance of embryonic stem cells using a novel human endometrial cell line. J Assist Reprod Genet. 2013; 30(5): 609-15. 
20. Wang D, et al. Immunohistochemistry in the evaluation of neovascularization in tumor xenografts. Biotech Histochem. 2008; 83(3-4): 179-89.

21. Joyce JA, Pollard JW. Microenvironmental regulation of metastasis. Nat Rev Cancer, 2009; 9(4): 23952.

22. Tuna B, et al. Association of mast cells with microvessel density in renal cell carcinomas. Eur Urol. 2006; 50(3): 530-4.

23. Jones $\mathrm{J}$, et al. Gene signatures of progression and metastasis in renal cell cancer. Clin Cancer Res. 2005; 11(16): 5730-9.

24. Li W, et al. Microarray profiling of human renal cell carcinoma: identification for potential biomarkers and critical pathways. Kidney Blood Press Res. 2013; 37(4-5): 506-13.

25. Kolenda T, et al. The mystery of let-7d - a small RNA with great power. Contemp Oncol (Pozn). 2014; 18(5): 293-301.

26. Chevrier S, et al. An Immune Atlas of Clear Cell Renal Cell Carcinoma. Cell. 2017; 169(4): 736749.e18.

27. Yang Z, et al. Infiltrating macrophages increase RCC epithelial mesenchymal transition (EMT) and stem cell-like populations via AKT and mTOR signaling. Oncotarget. 2016; 7(28): 44478-44491.

28. Toge $\mathrm{H}$, et al. Angiogenesis in renal cell carcinoma: the role of tumor-associated macrophages. Int $\mathrm{J}$ Urol. 2009; 16(10): 801-7.

29. Fischer C, et al. Anti-PIGF inhibits growth of VEGF(R)-inhibitor-resistant tumors without affecting healthy vessels. Cell. 2007; 131(3): 463-75.

30. Li C, et al. Knockdown of VEGF receptor-1 (VEGFR-1) impairs macrophage infiltration, angiogenesis and growth of clear cell renal cell carcinoma (CRCC). Cancer Biol Ther. 2011; 12(10): 872-80.

31. Tan HY, et al. Autophagy-induced RelB/p52 activation mediates tumour-associated macrophage repolarisation and suppression of hepatocellular carcinoma by natural compound baicalin. Cell Death Dis. 2015; 6: e1942.

32. Jin $\mathrm{H}$, et al. Targeting lipid metabolism to overcome EMT-associated drug resistance via integrin beta3/FAK pathway and tumor-associated macrophage repolarization using legumain-activatable delivery. Theranostics. 2019; 9(1): 265-278.

33. Ohba $\mathrm{K}$, et al. High expression of Twist is associated with tumor aggressiveness and poor prognosis in patients with renal cell carcinoma. Int J Clin Exp Pathol. 2014; 7(6): 3158-65.

34. Lee JH, et al. BMP-6 in renal cell carcinoma promotes tumor proliferation through IL-10-dependent M2 polarization of tumor-associated macrophages. Cancer Res. 2013; 73(12): 3604-14.

35. Barros $\mathrm{MH}$, et al. Macrophage polarisation: an immunohistochemical approach for identifying $\mathrm{M} 1$ and M2 macrophages. PLoS One. 2013; 8(11): e80908.

36. Rakaee M, et al. Prognostic Value of Macrophage Phenotypes in Resectable Non-Small Cell Lung Cancer Assessed by Multiplex Immunohistochemistry. Neoplasia. 2019; 21(3): 282-293. 
37. Ichimura T, et al. Prognostic significance of CD204-positive macrophages in upper urinary tract cancer. Ann Surg Oncol. 2014; 21(6): 2105-12.

38. Kawachi A, et al. Tumor-associated CD204(+) M2 macrophages are unfavorable prognostic indicators in uterine cervical adenocarcinoma. Cancer Sci. 2018; 109(3): 863-870.

39. Wittke F, et al. Interleukin 10 (IL-10): an immunosuppressive factor and independent predictor in patients with metastatic renal cell carcinoma. Br J Cancer. 1999; 79(7-8): 1182-4.

40. Zhao S, et al. Serum IL-10 Predicts Worse Outcome in Cancer Patients: A Meta-Analysis. PLoS One. 2015; 10(10): e0139598.

41. Daurkin I, et al. Tumor-associated macrophages mediate immunosuppression in the renal cancer microenvironment by activating the 15-lipoxygenase-2 pathway. Cancer Res. 2011; 71(20): 6400-9.

42. Roszer T. Understanding the Mysterious M2 Macrophage through Activation Markers and Effector Mechanisms. Mediators Inflamm. 2015; 2015: 816460.

43. Liu CY, et al. M2-polarized tumor-associated macrophages promoted epithelial-mesenchymal transition in pancreatic cancer cells, partially through TLR4/IL-10 signaling pathway. Lab Invest. 2013; 93(7): 844-54.

44. Hallett MA, et al. Cytokine stimulation of epithelial cancer cells: the similar and divergent functions of IL-4 and IL-13. Cancer Res. 2012; 72(24): 6338-43.

45. Terabe M, et al. NKT cell-mediated repression of tumor immunosurveillance by IL-13 and the IL-4RSTAT6 pathway. Nat Immunol. 2000; 1(6): 515-20.

46. Fujisawa $T$, et al. IL-13 regulates cancer invasion and metastasis through IL-13Ralpha2 via ERK/AP-1 pathway in mouse model of human ovarian cancer. Int J Cancer. 2012; 131(2): 344-56.

47. Gabitass RF, et al. Elevated myeloid-derived suppressor cells in pancreatic, esophageal and gastric cancer are an independent prognostic factor and are associated with significant elevation of the Th2 cytokine interleukin-13. Cancer Immunol Immunother. 2011; 60(10): 1419-30.

48. Chang Y, et al. Expression of IL-4 and IL-13 predicts recurrence and survival in localized clear-cell renal cell carcinoma. Int J Clin Exp Pathol. 2015; 8(2): 1594-603.

49. Satoh T, et al. The Jmjd3-Irf4 axis regulates M2 macrophage polarization and host responses against helminth infection. Nat Immunol. 2010; 11(10): 936-44.

50. Gao S, et al. Curcumin induces M2 macrophage polarization by secretion IL-4 and/or IL-13. J Mol Cell Cardiol. 2015; 85: 131-9.

\section{Figures}


A

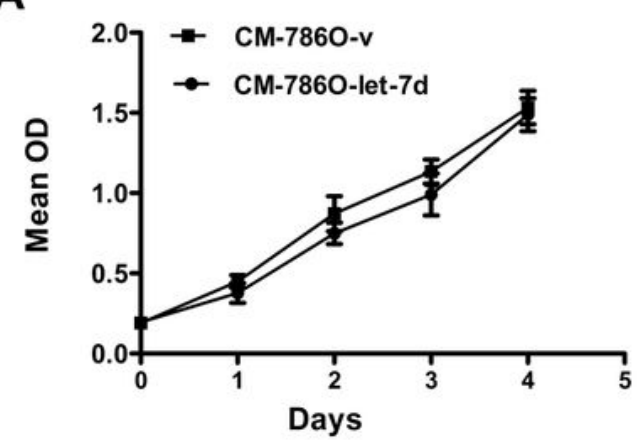

C

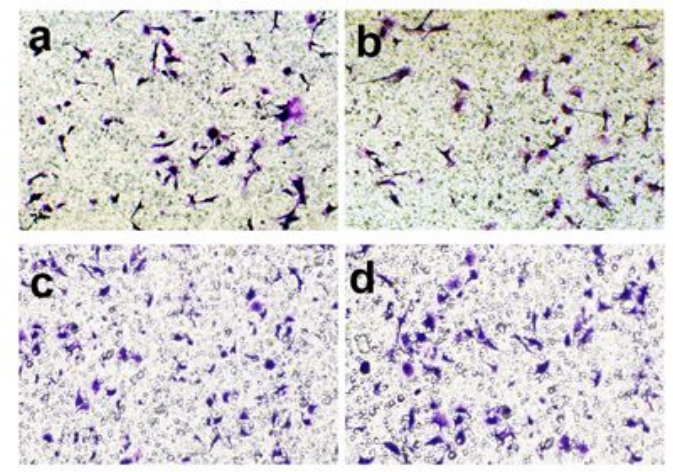

E

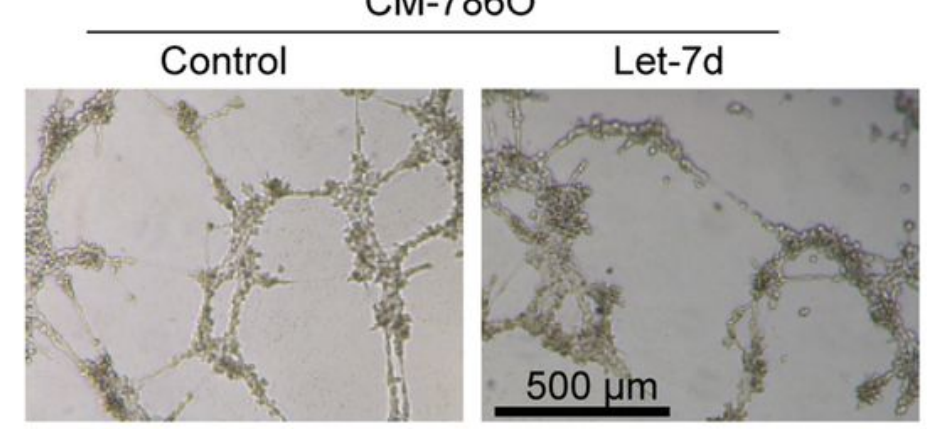

$\mathbf{F}$

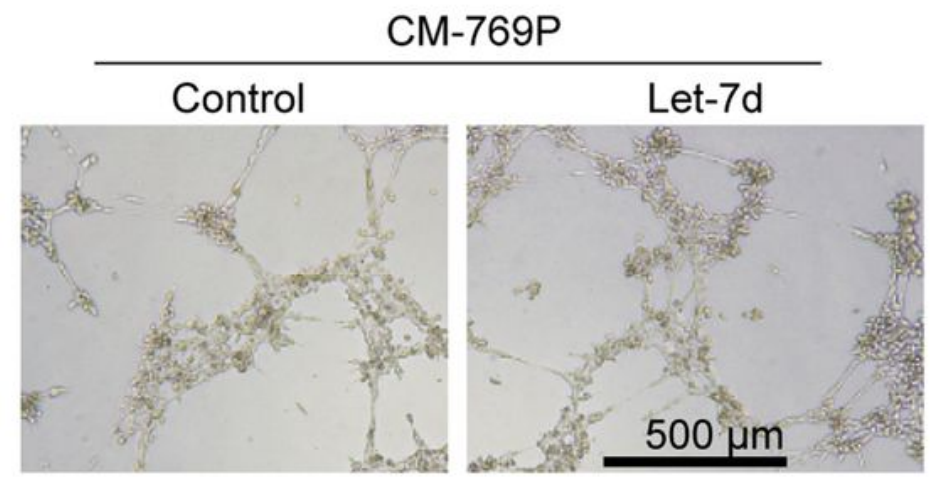

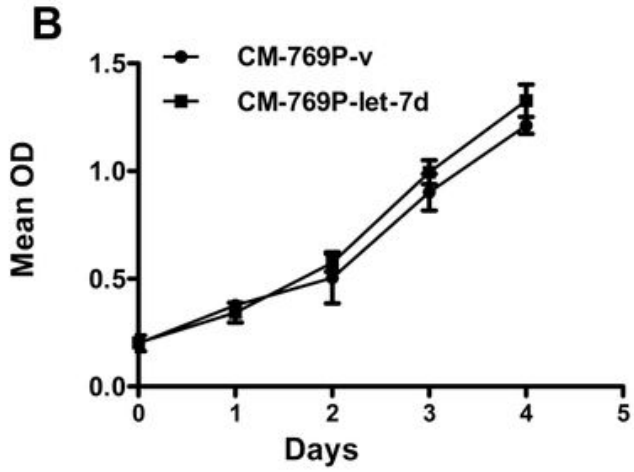

D

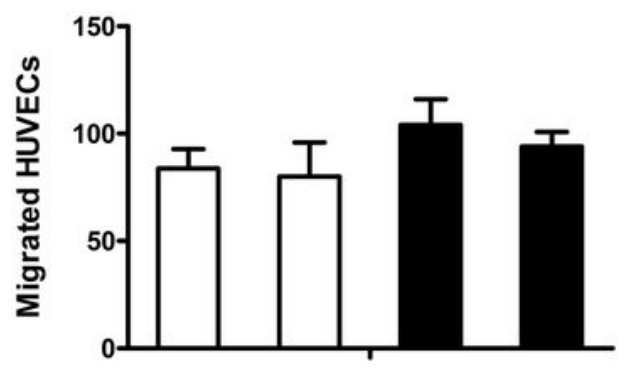

7860-v 7860-let-7d 769P-v 769P-let-7d
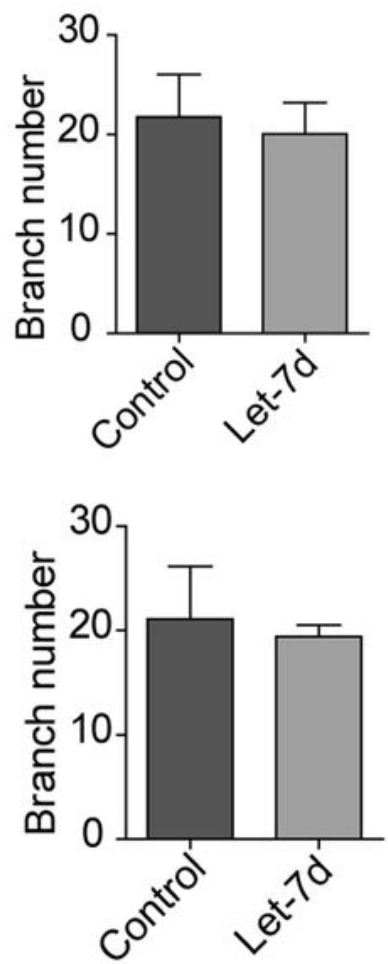

\section{Figure 1}

The conditioned medium from let-7d-overexpressing RCC cells had no effects on the proliferation, migration and tube formation of HUVECs $(A, B)$ The cell proliferation assay showed that conditioned medium from let-7d transfected 7860 and 769P cells has no significant influence on proliferation of HUVEC cells compared to that from vehicle control transfected cells. The data represent the mean \pm SD of three independent experiments with triplicates of each group. (C, D) Boyden chamber assays were 
performed, showing that HUVEC cell migration was not significantly different in conditioned medium from let-7d overexpressing 7860(b) and 769P(d) cells compared to that in conditioned medium from respective control cells $(a, c)$. Original magnification: $\times 20 .(E, F)$ Tube formation assay showed that the number of tubes formed by HUVEC cells in conditioned medium from let-7d overexpressing 7860 and $769 \mathrm{P}$ cells was not significantly different from those in conditioned medium from respective control cells. Bar, $500 \mu \mathrm{m}$. The quantification results of migrated cells and branch number are plotted in the right. Results are displayed as the mean \pm SD of three independent experiments with five random fields counted for each chamber/well. Different numbers between two groups were analyzed by two-tailed Student's t test. ${ }^{\star} p<0.05$. 
A

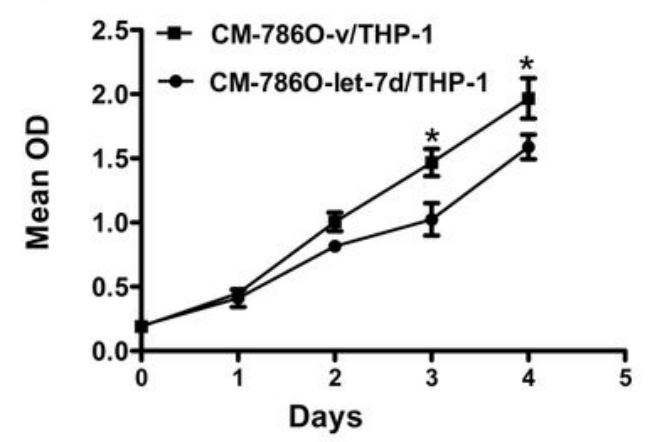

C

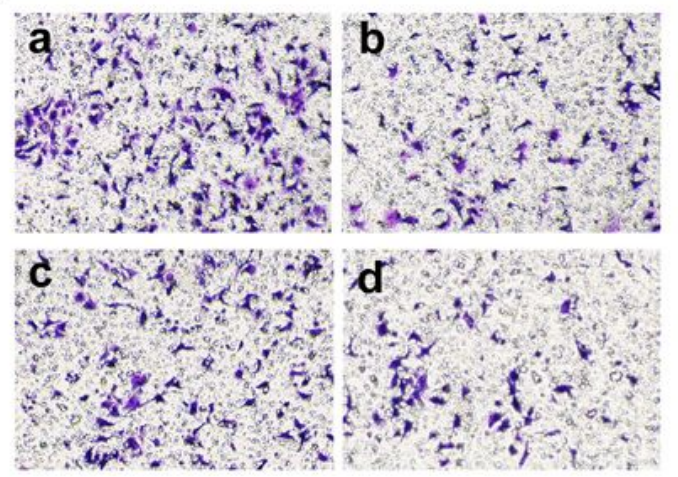

B

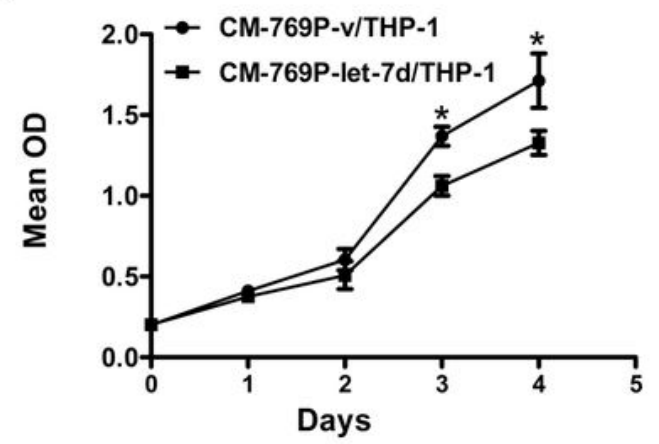

D

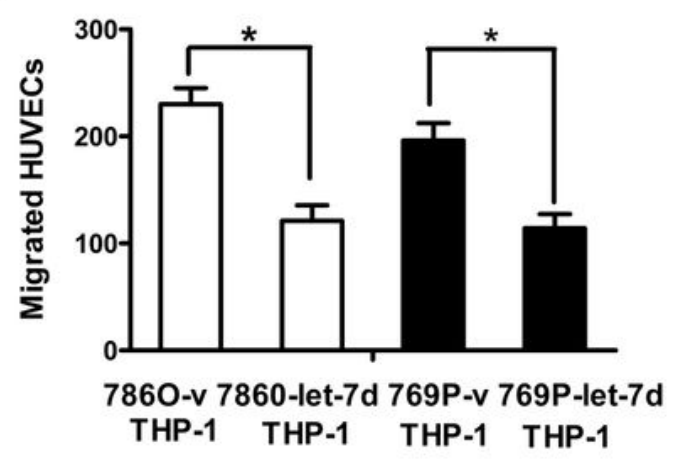

E
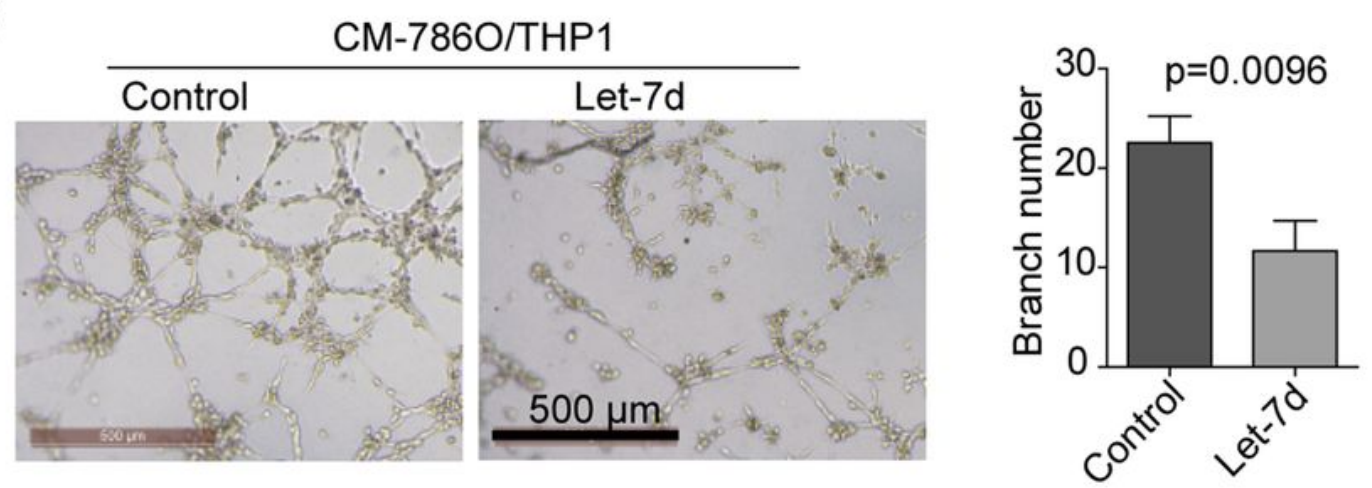

$\mathbf{F}$

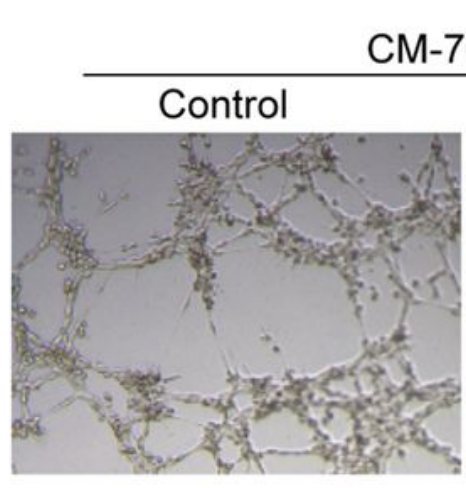

CM-769P/THP1
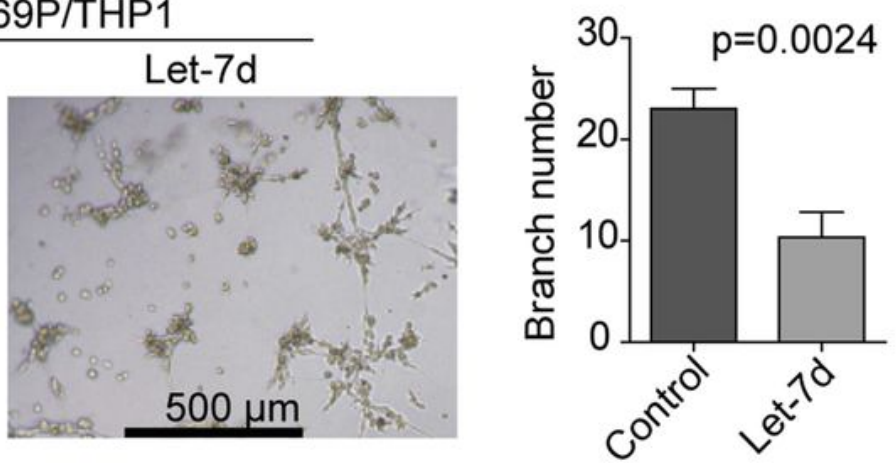

\section{Figure 2}

The conditioned medium from THP-1 cells cocultured with let-7d overexpressing RCC cells inhibited the proliferation, migration, and tube formation of HUVEC cells $(A, B)$ The cell proliferation assay showed that the proliferation of HUVEC cells in conditioned medium from cocultured system with let-7d transfected 7860 and 769P cells was significantly decreased compared to those in conditioned medium from cocultured system with respective vehicle control transfected cells. The data represent the mean \pm SD of 
three independent experiments with triplicates of each group. (C, D) Boyden chamber assays were performed, showing that HUVEC migration was significantly decreased in conditioned medium from cocultured system with let-7d transfected 7860 and $769 \mathrm{P}$ cells(b, d)when compared to system with respective control cells $(a, c)$. Original magnification: $\times 20$. $(E, F)$ Tube formation assay showed that the number of tubes formed by HUVEC cells in conditioned medium from cocultured system with let-7d transfected 7860 and 769P cells $(b, d)$ when compared to system with respective control cells $(a, c)$. Bar, $500 \mu \mathrm{m}$. The quantification results of migrated cells and number of tubes are plotted in the right. Results are displayed as the mean \pm SD of three independent experiments with five random fields counted for each chamber/well. Different numbers between two groups were analyzed by two-tailed Student's $t$ test. ${ }^{*} \mathrm{p}<0.05$. 
A

7860/THP1
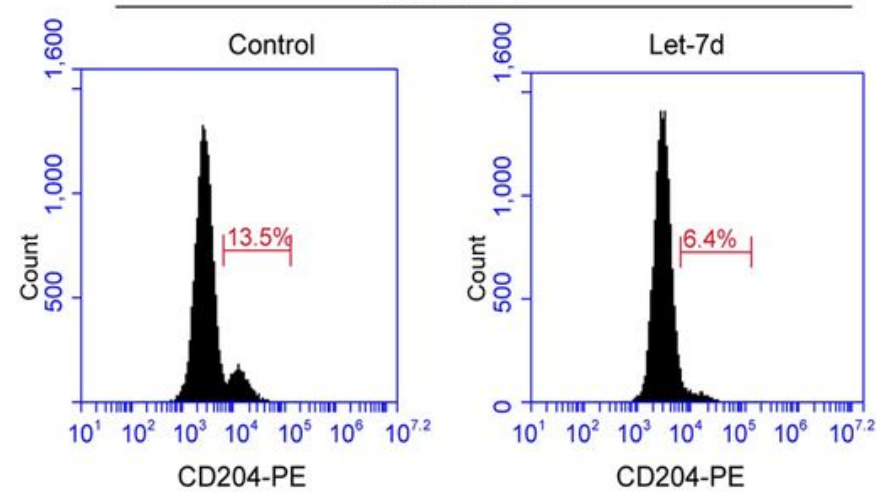

C

7860/THP-1

B

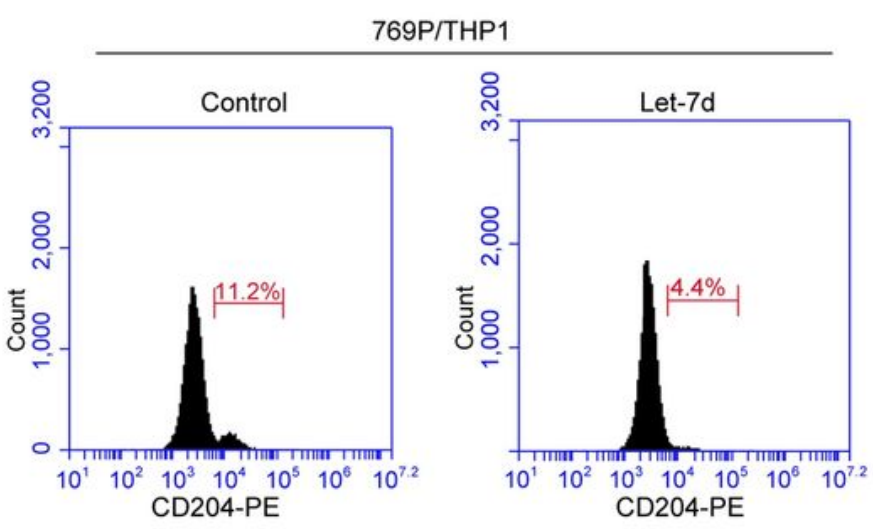

D

769P/THP-1
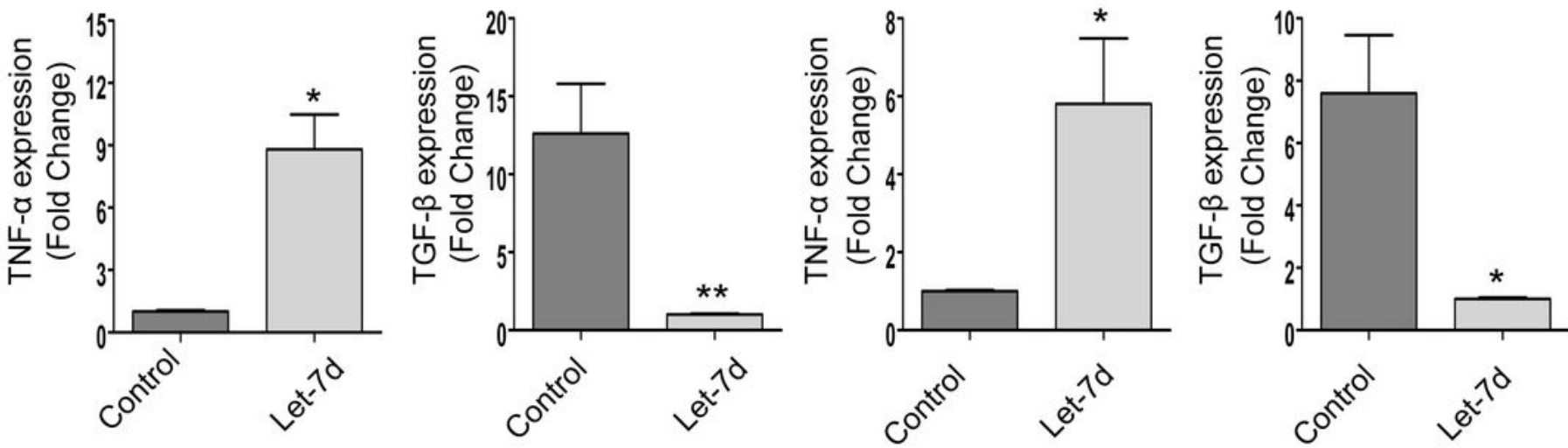

E

7860/THP-1

F

769P/THP-1
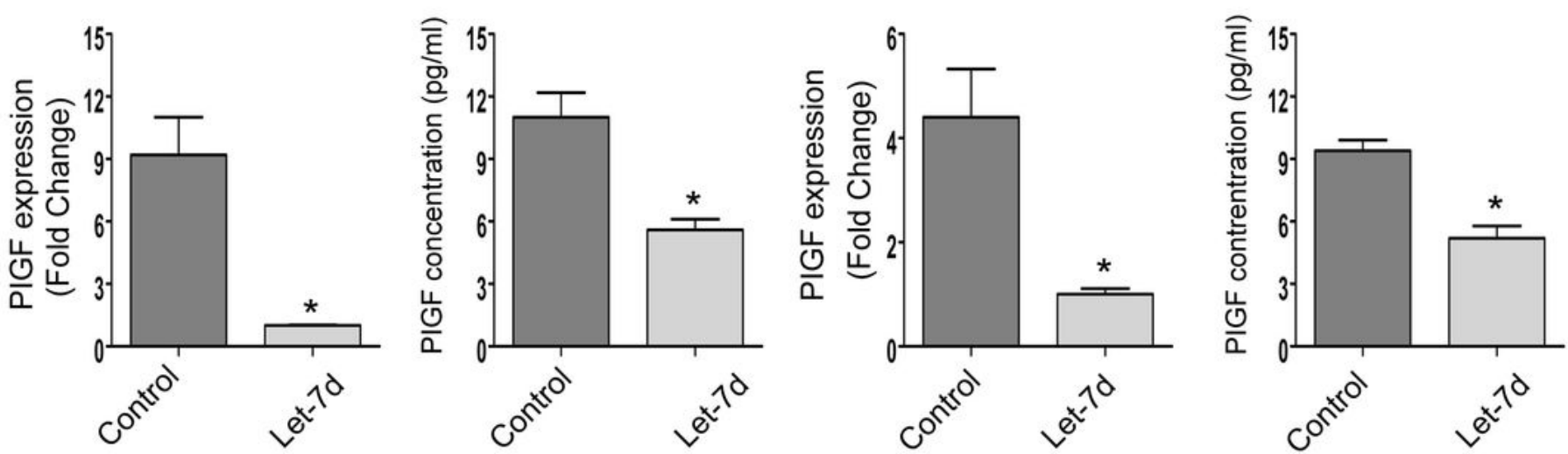

Figure 3

Let-7d overexpression in RCC cells inhibited the M2 polarization of THP-1 cells cocultured with RCC cells. (A, B) Flow cytometry analysis shows the percentage of CD204 (marker for M2 macrophage) positive cells were lower in M0 (THP-1 treated with 200 nM PMA for 48 hours)cells co-cultured with let-7d overexpressing RCC cells, as compared with those co-cultured with respective control RCC cells or cultured alone. (C, D) Real-time RT-PCR results show that M0 cells co-cultured with control 7860 and 
769P cells expressed low levels of pro-inflammatory cytokine TNF-a as well as high level of the antiinflammatory cytokine TGF- $\beta$, compared with M0 cells co-cultured with let-7d overexpressing 7860 and 769P cell. The mRNA expression was normalized to GAPDH mRNA. (E, F) The expression of PIGF was significantly decreased in M0 macrophages cocultured with let-7d overexpressing RCC cells, compared with those cocultured with control RCC cells, at mRNA level and protein level as detected by real time RTPCR (E) and ELISA assay (F). The data represent the mean \pm SD of three independent experiments with triplicates of each sample. Student's t test. ${ }^{*} p<0.05$.
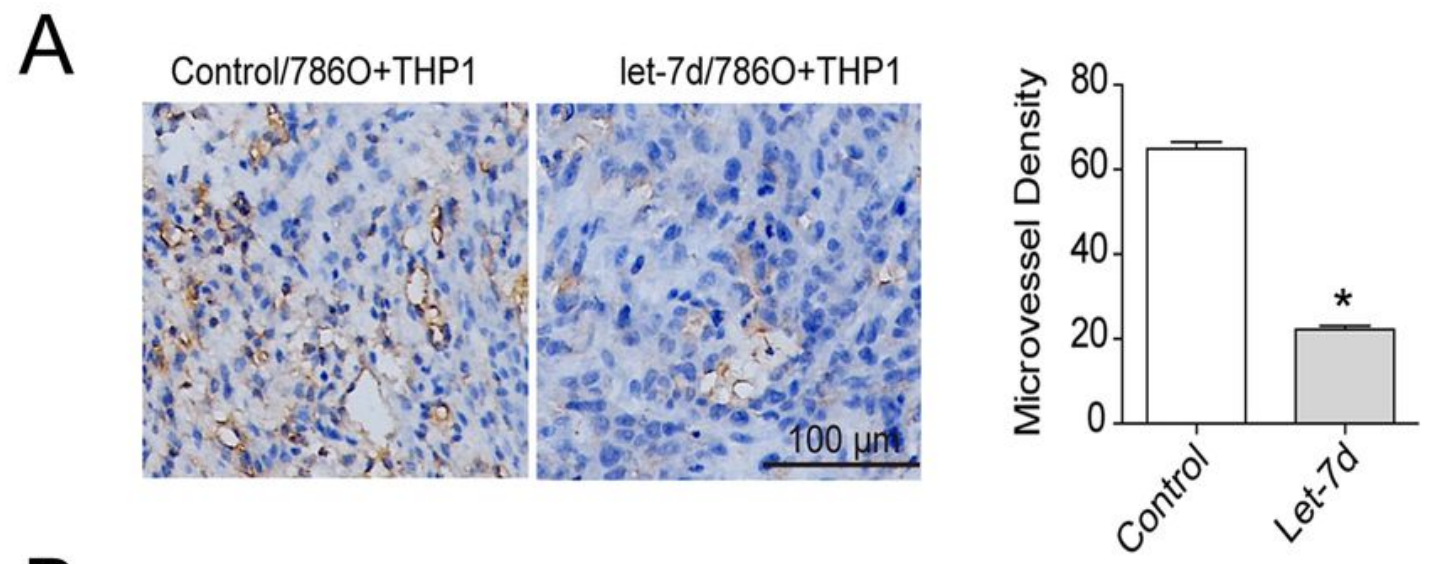

B

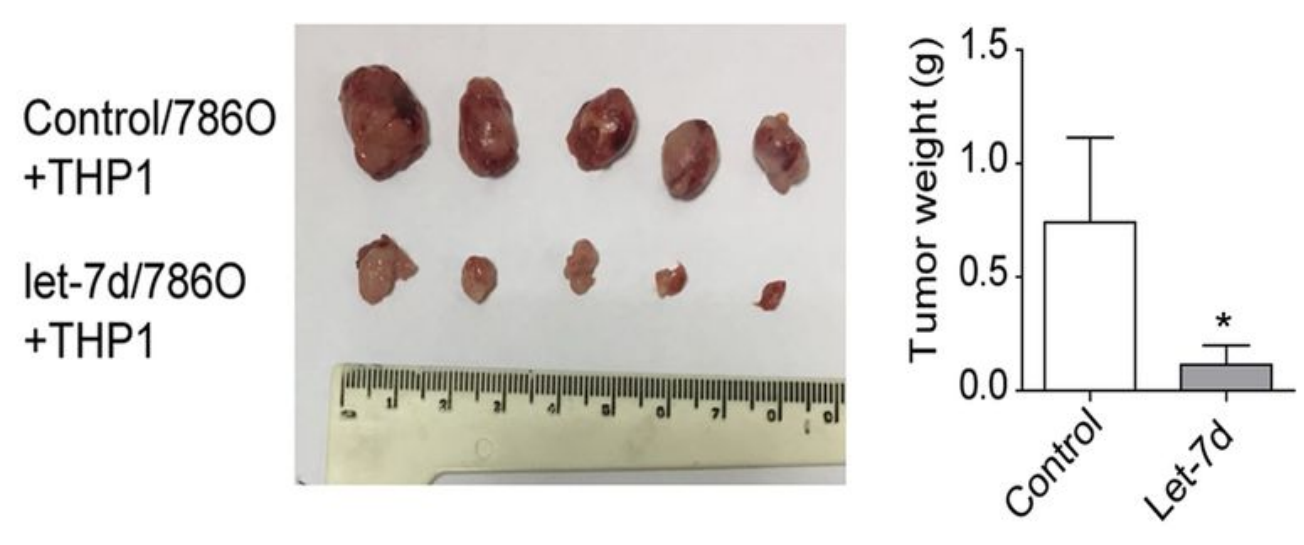

C
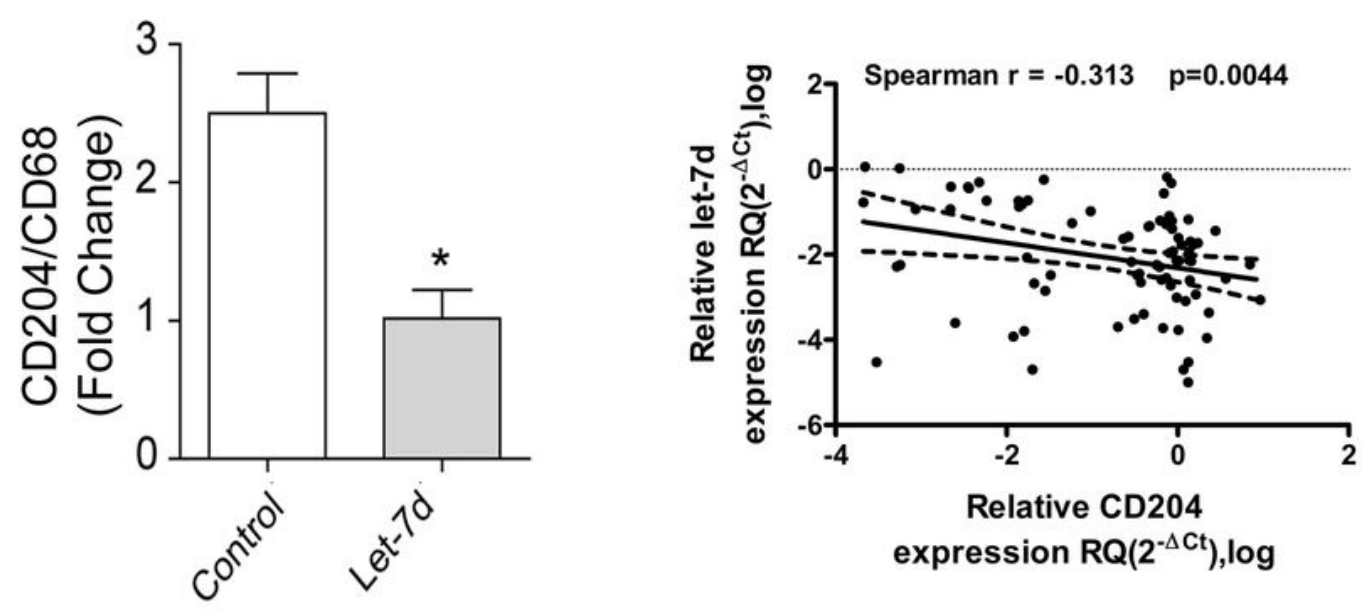


\section{Figure 4}

Let-7d overexpression inhibited macrophage M2 polarization and tumor angiogenesis in vivo. (A) Representative microphotograph of $\mathrm{IHC}$ staining shows the microvessels (CD31 positive) in subcutaneous xenograft formed by inoculating let-7d overexpressing 7860 cells or control 7860 cells together with $\mathrm{M} 0$ macrophages. Bar, $100 \mu \mathrm{m}$. Three random sections for each xenograft were subjected to IHC staining and calculation of MVD. Quantitative data of the mean MVD in each group were compared. (B) Photograph of subcutaneous xenograft formed by inoculating let-7d overexpressing 7860 cells or control 7860 cells together with M0 macrophages. Quantitative data of the mean tumour weight were shown in the right. (C) Real-time RT-PCR quantification of relative CD204 expression to CD68 expression in let-7d overexpressing groups was significantly lower than that in the control groups. The mRNA expression was normalized to GAPDH mRNA. Data represent the mean \pm SD of five mice. $p$ values in $(A-C)$ were obtained by two-tailed Student's t-test. (D) A linear regression and correlation among data from qRTPCR of let-7d and the mRNA level of CD204 is shown with r (spearman) and P-values indicated. Expression status is shown in a log scale. 
A

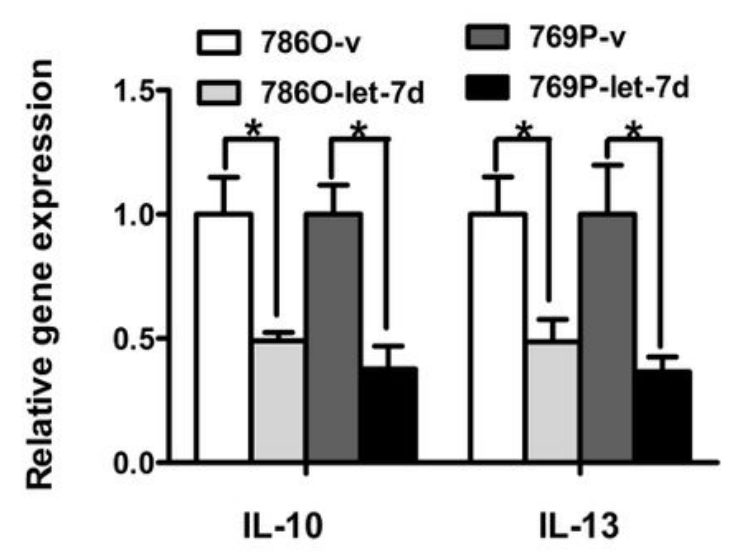

C

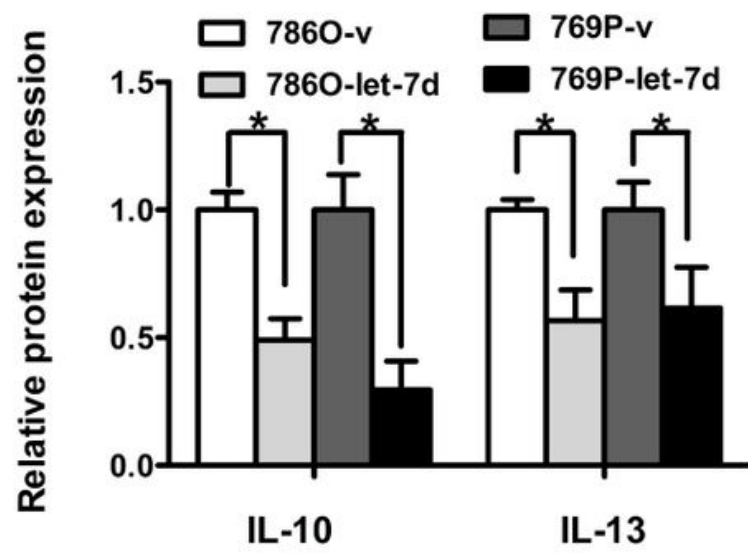

$E$

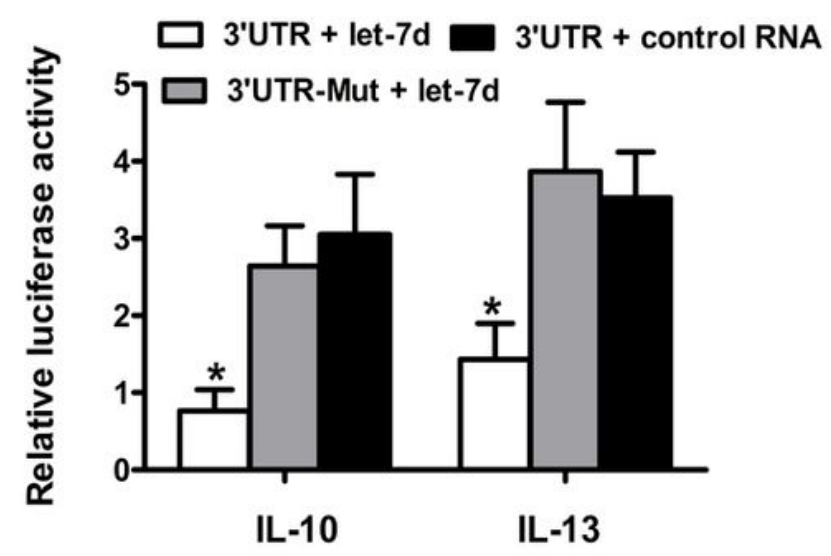

B

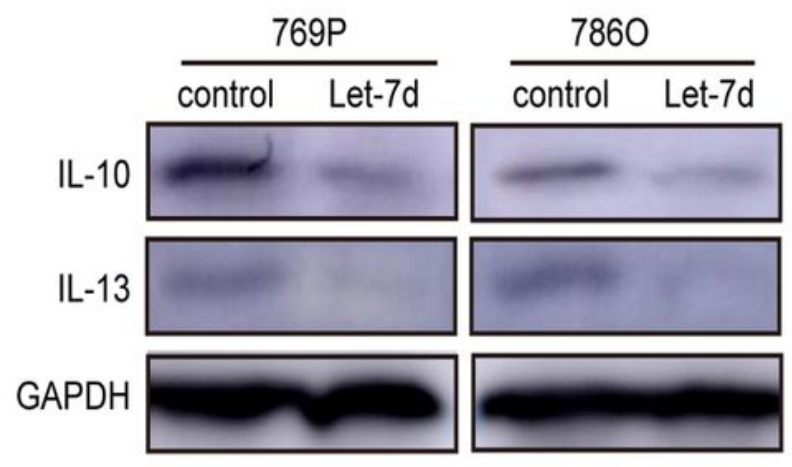

Mut 5' ...AUUUAUUACCUCUGAGCAAGAAA... Position 140-146 of IL10 wt 5' ...AUUUAUUACCUCUGAUACCUCAA... I I I I I I hsa-let-7d-5p 3' UUGAUACGUUGGAUGAUGGAGA

Mut $5 '$ '...GUGUACAGAAUUCUGAGCAAGAA... Position 567-574 of IL13 wt 5' ...GUGUACAGAAUUCUGCUACCUCA... 1 I 1 I I I I hsa-let-7d-5p $\quad 3^{\prime} \quad$ UUGAUACGUUGGAUGAUGGAGA

Figure 5

IL-13 and IL-10 were direct target genes of let-7d in RCC cells (A) The expression of endogenous IL-10 and IL-13 was inhibited in the let-7d overexpressing 7860 and 769P cells, compared with the control, at mRNA level as detected by real time RT-PCR; (B) Representative Western blot results show that the protein level of IL-10 and IL-13 were also downregulated following lenti-pri-let-7d infection. GAPDH served as an internal loading control. The corresponding densitometry of each band is presented in a bar graph in (C). 
(D) Sequence alignment of human let-7d seed sequence with the 3'-UTRs of IL-10 and IL-13. The mutated sequence in the putative let-7d binding sites for each gene is shown above each gene set (Mut). (E) Luciferase activity of various reporter plasmids. Statistical significance was obtained using one way ANOVA. Data present in $(A, C, E)$ are the mean \pm SD of three independent experiments. ${ }^{*} p<0.05$.

A

CM-7860-let-7d/THP-1
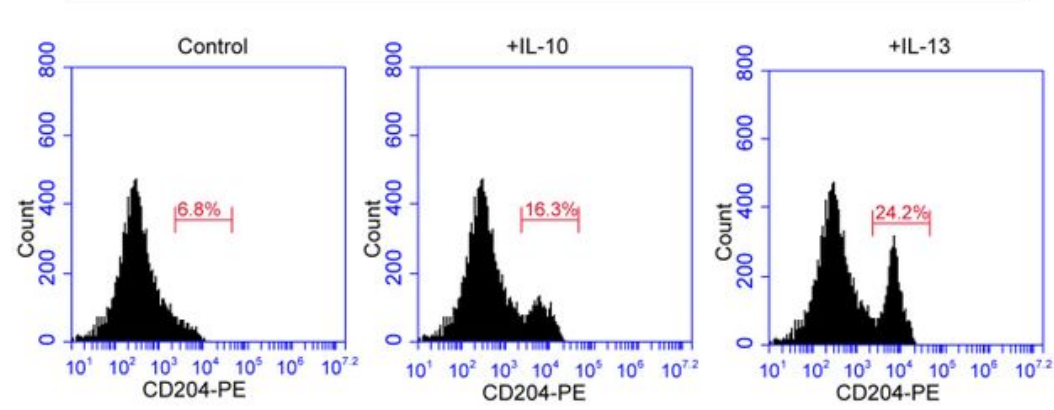

C

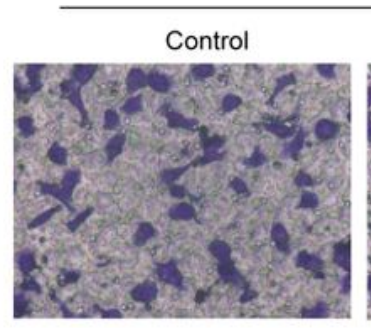

CM-7860-let-7d/THP-1
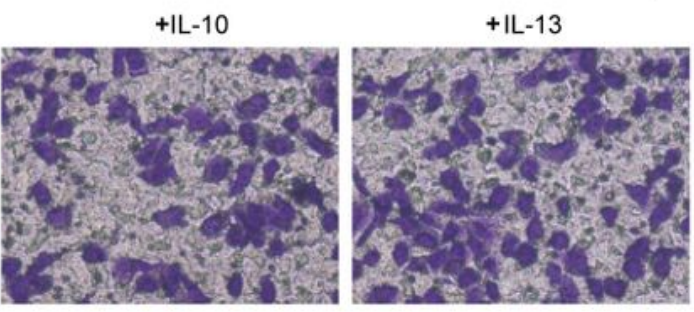

D

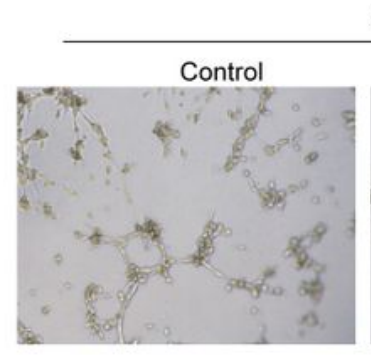

CM-7860-Let-7d / THP1
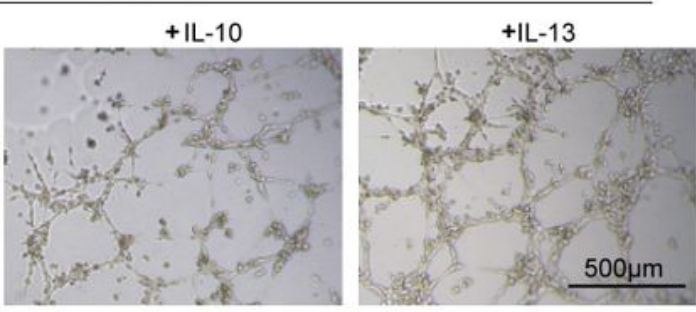

$\mathrm{F}$

E
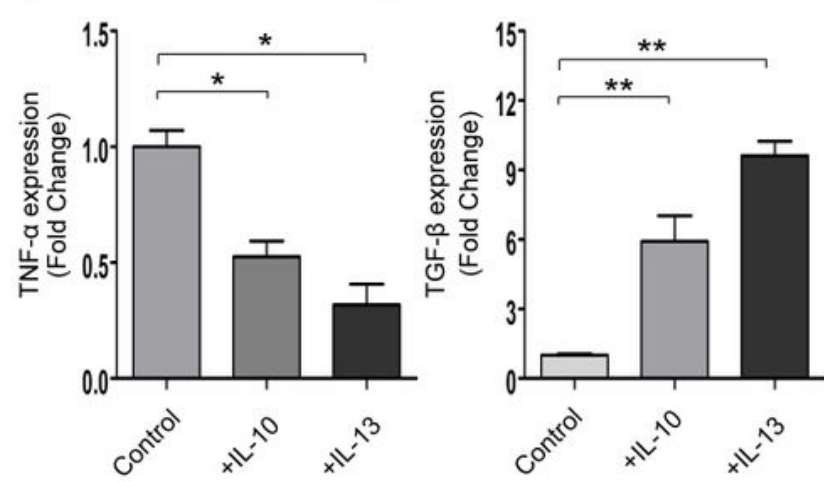

G

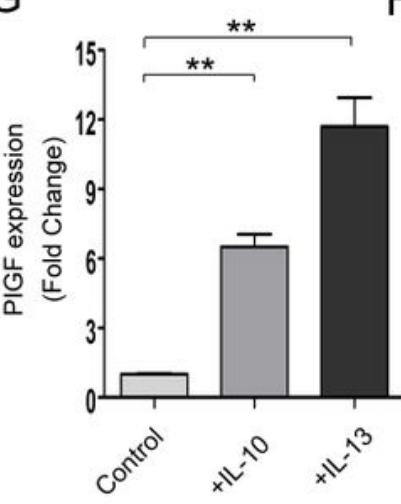

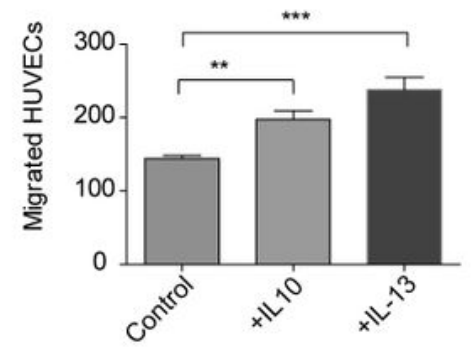
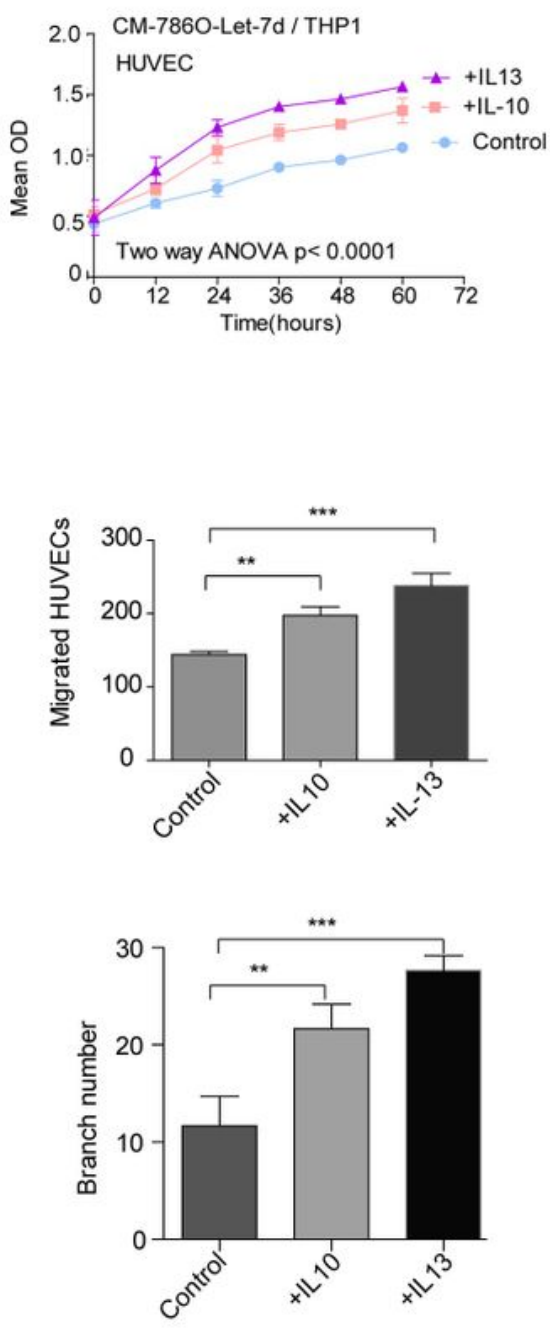

$\mathrm{H}$

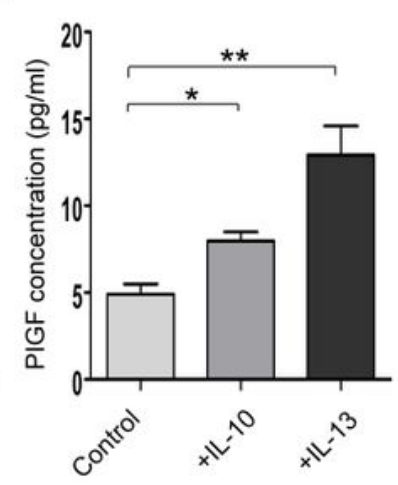

Figure 6 
Rescue of IL-10 and IL-13 eliminated the effects of let-7d in vitro. (A) Flow cytometry analysis shows the percentage of CD204 positive cells were restored in the presence of purified IL-10 or IL-13 $(100 \mathrm{ng} / \mathrm{ml})$ respectively, as compared with control (PBS, phosphate buffer solution). (B) The cell proliferation assay showed that the proliferation of HUVEC cells in conditioned medium from coculture system with let-7d overexpressing RCC cells in the presence of $100 \mathrm{ng} / \mathrm{ml} \mathrm{IL-10} \mathrm{or} \mathrm{IL-13} \mathrm{was} \mathrm{significantly} \mathrm{increased}$ compared with that in the presence of control. The data represent the mean \pm SD of three independent experiments with triplicates of each sample. ${ }^{*} p<0.05$. Statistical significance was obtained using two way ANOVA. (C) Represented micrographs and quantitative data of a Boyden chamber assay show that the migration of HUVEC cells was significantly restored in the conditioned medium from M0 macrophages cocultured with let-7d overexpressing RCC cells in the presence of $100 \mathrm{ng} / \mathrm{ml} \mathrm{IL-10} \mathrm{or} \mathrm{IL-13.} \mathrm{Original}$ magnification: $\times 40$. (D) Photograph shows that the tubes formed by HUVEC cells following the addition of IL-10 or IL-13 in the coculture system. Bar, $500 \mu \mathrm{m}$. Histogram displays the quantitative data of branch number formed by HUVEC cells in conditioned medium with IL-10, IL-13 or control respectively. (C-D) The data presented are the mean \pm SD of three independent experiments with five random fields counted for each chamber/well. (E, F) Real-time RT-PCR results show that the expression of pro-inflammatory cytokine TNF- $\alpha$ was significantly decreased (E) and the expression of anti-inflammatory cytokine TGF- $\beta$ was significantly increased (F) in M0 cells cocultured with let-7d overexpressing 7860 after addition of $100 \mathrm{ng} / \mathrm{ml}$ IL-10 or IL-13. The mRNA expression was normalized to GAPDH mRNA. $(G, H)$ The expression of PIGF was significantly decreased in conditioned medium from M0 macrophages cocultured with let-7d overexpressing 7860 cells in the presence of control, compared with those in the presence of $100 \mathrm{ng} / \mathrm{ml}$ IL-10 or IL-13, at mRNA level (G) and protein level $(\mathrm{H})$ as detected by real time RT-PCR and ELISA assay. The data represent the mean \pm SD of three independent experiments with triplicates of each sample. Student's $t$ test. ${ }^{*} \mathrm{p}<0.05$. 
A

IL-10

GAPDH
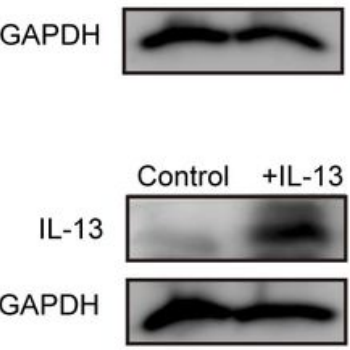

C
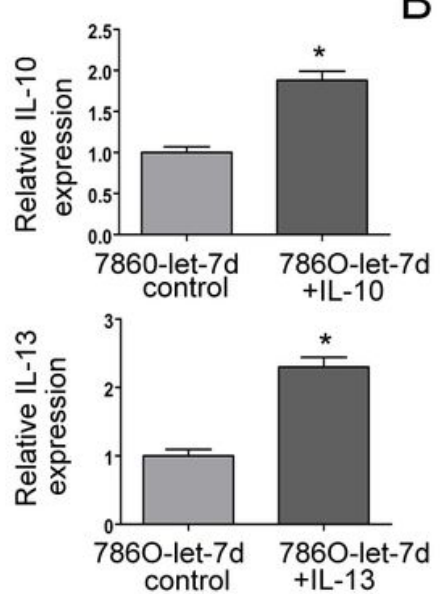
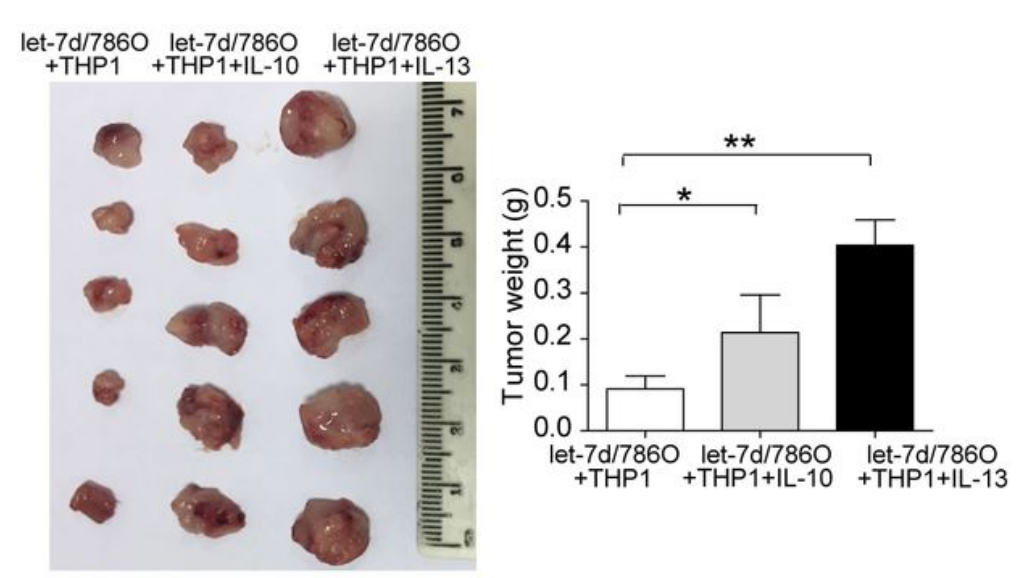

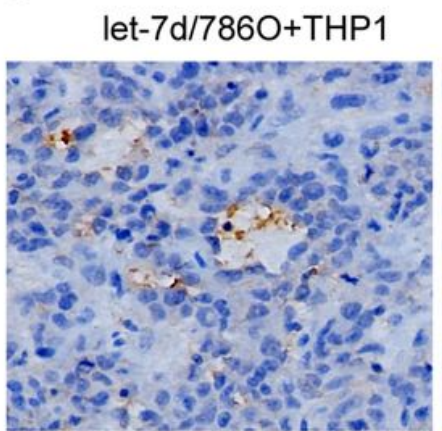

let-7d/786O+THP1+IL-10

let-7d/786O+THP1+IL-13
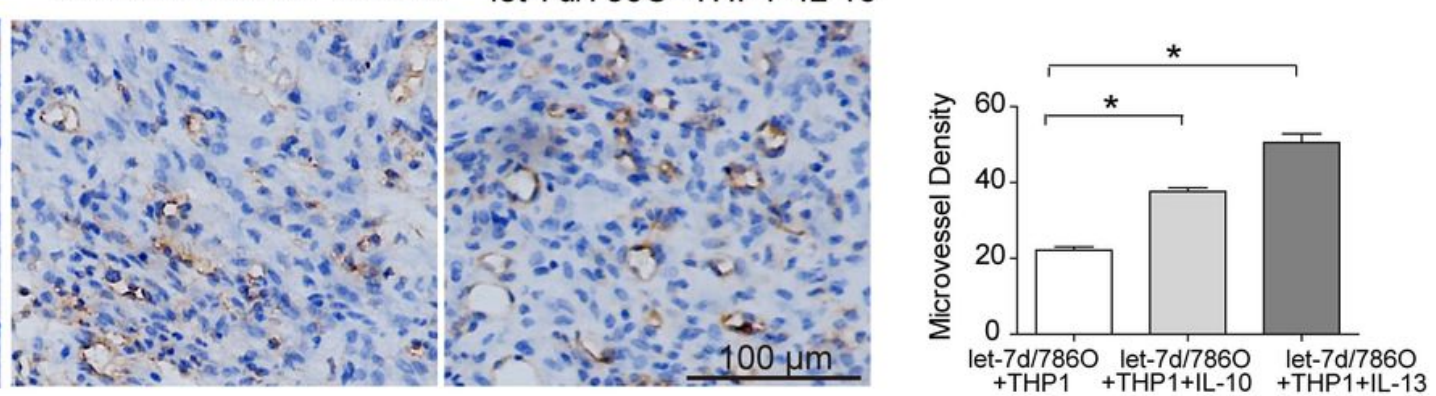

D

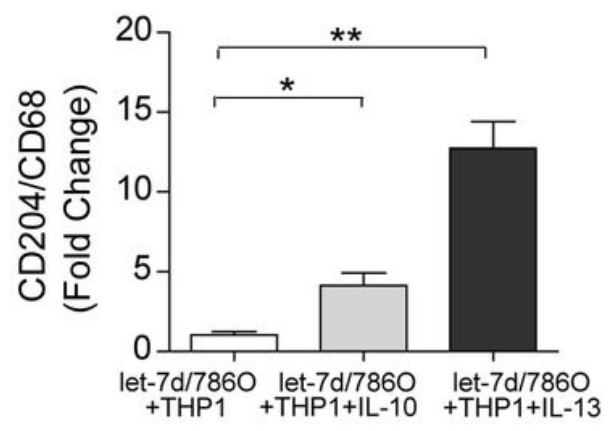

E

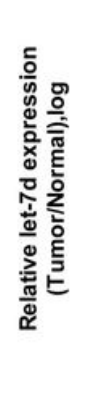

Spearman $r=-0.36 \quad P=0.001$

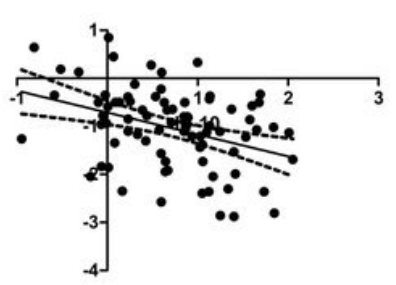

Relative IL-10 expression (Tumor/Normal),log
F

Spearman $r=-0.38 \quad P=0.0005$
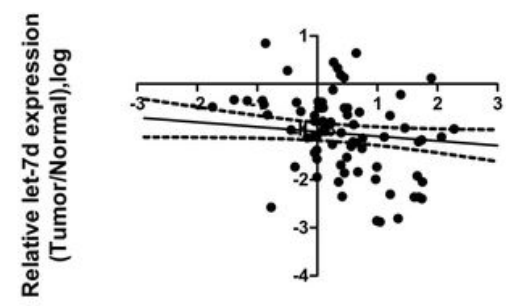

Relative IL-13 expression (Tumor/Normal),log

Figure 7

Rescue of IL-10 and IL-13 overcomes the effects of let-7d in vivo. Let-7d expression inversely correlated with the IL-10 and IL-13 mRNA levels in RCC tissues. (A)Western blot results demonstrate that the protein level of IL-10 and IL-13 were increased after the lenti-pri-let-7d 7860 cells were infected with lentivirus carrying the expression cassette without the 3'-UTRs of IL-10 or IL-13 respectively. The corresponding densitometry of each band is presented in a bar graph. The data represent the mean \pm SD of three independent experiments. (B) Photograph of subcutaneous xenografts from the three groups.

Quantitative data of the mean tumour weight were shown in the right. (C) Representative graphs of IHC staining of MVD (CD31 positive) in xenografts formed by inoculating M0 macrophages together with lent-

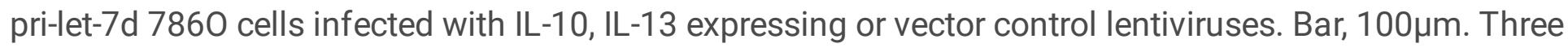
random sections for each xenograft were subjected to IHC staining and calculation of MVD. Quantitative data of the mean MVD in each group were compared in the right. (D) Real-time RT-PCR quantification of 
relative CD204 expression to CD68 expression in xenografts formed by inoculating M0 macrophages together with lent-pri-let-7d 7860 cells infected with IL-10, IL-13 expressing or vector control lentiviruses. The mRNA expression was normalized to GAPDH mRNA. Data represent the mean \pm SD of five mice. $P$ values were obtained by two-tailed Student's t-test. $(E, F)$ The relationship between let-7d expression and IL-10 (E) and IL-13 (F) mRNA levels in RCC samples (80 cases). Statistically significant reverse correlations were observed between let-7d and IL-10 and between let-7d and IL-13 mRNA levels using the two-tailed Spearman's test.

\section{Supplementary Files}

This is a list of supplementary files associated with this preprint. Click to download.

- figS1.tif

- supportinginformation.doc 